\title{
ALGA: Adaptive lexicon learning using genetic algorithm for sentiment analysis of microblogs
}

\author{
Hamidreza Keshavarz \\ Mohammad Saniee Abadeh ${ }^{1}$ \\ \{keshavarz.h, saniee\}@modares.ac.ir \\ Faculty of Electrical and Computer Engineering, Tarbiat Modares University, Tehran, Iran
}

\begin{abstract}
Sentiment analysis is about classifying opinions expressed in text. The aim of this study is to improve polarity classification of sentiments in microblogs by building adaptive sentiment lexicons. In the proposed method, corpora-based and lexicon-based approaches are combined and lexicons are generated from text. The sentiment classification is formulated as an optimization problem, in which the goal is to find optimum sentiment lexicons. A novel genetic algorithm is then proposed to solve this optimization problem and find lexicons to classify text. The algorithm generates adaptive sentiment lexicons, and then a meta-level feature is extracted based on it, which is then used alongside Bing Liu's lexicon and n-gram features. The experiments are conducted on six datasets. In terms of accuracy, the results outperform the state-of-the-art methods proposed in the literature in two of the datasets. Also, in four of the datasets, the proposed approach outperforms in terms of Fmeasure. Applying the proposed method on six datasets, the accuracy is higher than $80 \%$ in all six datasets and the F-measure is higher than $80 \%$ in four of these datasets. Using the sentiment lexicons created by the proposed algorithm, one can get a better understanding of the specific language and culture of Twitter users and sentiment orientation of words in different contexts. It is
\end{abstract}

${ }^{1}$ Corresponding Author

(C) 2017. This manuscript version is made available under the Elsevier user license http://www.elsevier.com/open-access/userlicense/1.0/ 
also shown that it is useful not to omit the conventional stop-words, as each word can have its sentimental implications.

Keywords: Sentiment Analysis, Genetic Algorithm, Twitter, Sentiment Lexicon, Social Media, Evolutionary Computation

\section{Introduction}

Every day, social media users express their feelings towards various subjects such as politicians, governmental organizations, and consumer products [1], creating an enormous amount of data. One of the most popular social networks is Twitter, with 320 million monthly active users who post about 500 million Tweets (up to 140 characters) per day². Processing and analyzing this rich, yet easily available resource enables us to create actionable knowledge to be used in decision making process [2].

Sentiment analysis or opinion mining is a discipline which deals with analyzing and classifying subjective opinions, sentiments and emotions of people towards products, organizations, individuals and other topics [1, 3, 4], expressed in text, such as tweets [5], reviews [6], forums [7], blogs [8] and news [9]. Sentiment analysis makes it possible to capture the trends of people [10]. Lexicon-based and corpus-based methods are two main approaches for sentiment classification of documents. A lexicon-based method uses a sentiment lexicon which includes sentiment words and phrases with assigned numeric scores. These scores reveal if sentiment phrases are positive or negative, their intensity, and/or their emotional orientations. A corpus-based method trains a classifier on a labelled corpus [11].

The proposed method concerns detecting polarity by sentiment analysis of Twitter data and classifying the polarity of tweets into positive and negative classes. A standard approach for

\footnotetext{
${ }^{2}$ https://about.twitter.com/company
} 
polarity detection of the text is using existing sentiment lexicons to classify text [12-15]. However, these lexicons are usually static which make them less suitable to be applied in polarity detection on tweets, due to challenges of sentiment analysis on Twitter data. These challenges include prevalent misspellings and slangs which develops Twitter's own culture and language [16], wide range of topics covered by Twitter users (unlike in the case of news and online journals) [16], and short length of tweets which limits their sentiment cues [17]. Furthermore, creation of manual lexicons is hard [12]. This barrier is overcome by combining the two sentiment classification methods. Including all frequent words as sentiment words, the proposed method generates dynamic lexicons based on corpus, and then classifies text using these lexicons.

The main contributions are as follows: (i) The problem of finding optimal dynamic lexicons for sentiment analysis is modelled as an optimization problem; (ii) To tackle this optimization problem, a genetic algorithm is proposed with novel penalty and reward mechanisms, which yields better convergence and outperforms previous methods on most assessed datasets; and (iii) By considering every word, including stop-words as sentiment words, it is shown that generating an adaptive lexicon can lead to a better understanding of sentiment phrases and capturing the delicacies of twitter users' language.

An evaluation of the proposed method on six Twitter datasets is presented. The results outperform the best results in two of datasets in terms of accuracy and four datasets in terms of F-measure. Furthermore, it is demonstrated that each word, even stop-words in a text can be considered as a sentiment word, as shown in some previous studies. The proposed method uses the created lexicon features alongside another lexicon and n-gram features to improve accuracy and F-measure. It yields results better than the state-of-the-art methods (subsection 2.1). It also outperforms the accuracy and F-measure of the best reported result on one of the datasets (Strict OMD) by more than five percentage points. Likewise, the positive class F-measure values are better than other results in five datasets. The negative F-measure value outperforms the other methods in four 
datasets. Furthermore, it is demonstrated that a random search algorithm for creating a sentiment lexicon does not perform well on these datasets, yielding an F-measure value of around 50 percent on all of the datasets.

The remainder of the paper is organized as follows: In section 2, the related works are provided. In section 3, the proposed method is explained. Experimental results are discussed in section 4. Section 5 concludes the paper and future works are presented.

\section{Related Work}

In this section, the related work for sentiment analysis in the field of microblogs, and sentiment lexicons are assessed. Furthermore, the works that use evolutionary algorithms in sentiment analysis are reviewed. Since the proposed method generates dynamic sentiment lexicons, existing sentiment lexicons are assessed as well. The proposed work is about classifying tweets, and hence, the works done on this media outlet are reviewed. Since in this method, a genetic algorithm is incorporated for generating lexicons, the works done in the field of evolutionary algorithms in sentiment analysis are studied as well.

\subsection{Twitter Sentiment Analysis}

Since tweets cannot exceed 140 characters, they are usually straightforward and are a great resource for opinion mining [18]. Since it is hard to manually label data for polarity classification of tweets, some studies use emoticons as labels [19-21]. In [18] it is argued that these methods can generate noise.

Go et al. [20] collected a Twitter dataset with more than 1,600,000 tweets and labelled it using emoticons. Liu et al. in [21] also created a dataset and then labelled the tweets with a combination of using emoticons and manual labelling. Da Silva et al. [16] proposed using classifier ensembles for Twitter sentiment classification. 
Some methods combine using of lexicons and learning based techniques for sentiment classification, such as [22-25]. Hu et al. in [26] incorporated the networked data to use emotional spread for sentiment classification. In [27], features based on semantic concepts are added to the training set. In [28], another approach that uses meta-level features for Twitter sentiment classification is used. In this method, different dimensions of words are employed for subjectivity and polarity classification. Kaewpitakkun et al. built an add-on lexicon that calculates scores for objective and out of vocabulary words, and used a weighting scheme for features [29]. Saif et al. proposed an adaptation method for sentiment lexicons to capture the meanings of words in different contexts in Twitter [30]. The proposed method in this paper similarly tries to generate an adaptive sentiment lexicon. Coletta et al. in [31] used an SVM classifier with a cluster ensemble to classify tweets. Lu [32] used microblog-microblog relations, incorporating social relations and text similarities, for building a semi-supervised classifier. Saif et al. [33] used an approach for capturing patterns of words, and evaluate it on tweet-level and entity-level sentiment analysis. They incorporated latent semantic relations for improving classification accuracy. Baecchi et al. [34] used a multimodal approach for feature learning for classification of tweets that may contain pictures. $\mathrm{Hu}$ et al. [35] incorporated emotional signals, such as emoticons, in an unsupervised learning framework in Twitter datasets. In [36], Bakliwal et al. used a sentiment scoring function for classification of tweets. Saif et al. [37] used a lexicon-based approach in which they calculated sentiment orientation and strength in Twitter. Finally, Wu et al. [38] incorporated social connections between tweets of the same author and social relations between users, to improve accuracy.

The proposed work uses meta-level features, as in [28]. It also creates dynamic sentiment lexicons, as in [36].

The works explained in this section yield promising results and are significant methods. However, there are some shortcomings that can be seen in them: (i) they use static lexicons, but twitter is a 
dynamic platform and meanings of words can change over time; (ii) in some methods, text features are used which increase the dimensionality of data significantly, and this is problematic, especially in ensemble methods; (iii) The words present in sentiment lexicons are scarcely used in tweets; (iv) The methods do not give an insight about words; and (v) they usually cannot detect irony.

\subsection{Sentiment Lexicons}

Sentiment lexicons are sets of words and phrases that are associated with numeric scores, which show the sentiment and/or emotional orientation of the words. In some lexicons, such as Bing Liu's lexicon [1], these words are simply labelled as positive or negative. The granularity of scores is higher in some lexicons, such as AFINN, in which the assigned scores range from -5 to +5 .

Wilson et al. [39] created the Opinion Finder lexicon in which the words are labelled as positive or negative. Another lexicon is ANEW, presented in [40], which is a lexicon with affective norms for English words. Nielsen created a lexicon named AFINN inspired from ANEW [41] for social networks, and included words prevalent in social media, such as "WTF" and "LOL" in it. In AFINN, the negative words have a score ranging from -5 to -1 , and positive words are in the range of +1 to +5. This lexicon contains 2477 words.

Esuli and Sebastiani [42], and Baccianella et al. [43] created the SentiWordNet lexicon which is based on WordNet. SentiStrength, created by Thelwall et al. [44], is another lexicon, which is used for strength detection. NRC, a lexicon for emotion mining was developed by Mohammad and Turney [45], in which words are associated with a label for their emotional scores. Liu [1] also created a lexicon which is known as Bing Liu's lexicon. This lexicon contains 2006 words labelled as positive and 4683 words labelled as negative.

Another lexicon, NRC Hashtag Sentiment Lexicon was automatically created using a collection of 775310 tweets with positive or negative hashtags; the tweets were labelled as positive or negative by their hashtag polarities, and the sentiment score was found using pointwise mutual information. 
Sentiment scores for each entry range from -5 to +5 . This lexicon was created by NRC-Canada team that won the SemEval task [46]. Sentiment140 Lexicon was also presented by NRC-Canada team, which uses positive and negative emoticons for labelling the sentiment words.

All of the aforementioned lexicons are created for English language. The approaches that use lexicons face challenges, such as dealing with negations [47]. The adaptive lexicons of ALGA are compared to AFINN, Sentiment140, NRC hashtag and Bing Liu's lexicon which have been used extensively in literature.

\subsection{Application of Evolutionary Computation in Sentiment Analysis}

In this section, the use of evolutionary algorithms in sentiment analysis is reviewed. Abbasi et al. [48] used a hybridized genetic algorithm for feature selection in opinion classification in web forums. In [49], a hybrid of support vector machines and particle swarm optimization is used for sentiment analysis of movie reviews. A tabu search enhanced markov blanket is used for sentiment extraction in [50]. In [51], a large set of syntactic, semantic and discourse level features are considered, and a genetic algorithm is used for selecting features that optimize the accuracy. In [52], an artificial immune system is incorporated for sentiment classification. The individual format they proposed is in binary format, in which each bit shows the presence of a word. In [53], PSO/ACO2, a hybrid of Particle Swarm Optimization and Ant Colony Optimization is used for finding whether a post contains specific arguments. In [54], the Arora et al. use genetic programming for constructing new evolved features based on existing features to improve accuracy. Govindarajan [55] uses a hybrid of Naive Bayes classifier and genetic algorithm for classification of movie reviews. Carvalho et al. [56] proposed a genetic algorithm for selecting paradigm words from a set of candidate words for sentiment classification of Tweets.

Since adaptive lexicons are created in this work, the trends in the ever-changing environment of Twitter can be captured. The lexicons built by the proposed method can be updated easily to adapt 
with the changes in the culture of Twitter users. Using only one feature (the ALGA feature), the results of the proposed method are promising. A static sentiment lexicon (Bing Liu's lexicon [1]) is also used alongside the stand-alone ALGA feature in another versions of the proposed method. The adaptive lexicons created by the proposed method and Bing Liu's lexicon complement each other and add to the accuracy measures.

\section{Adaptive Lexicon learning by Genetic Algorithm (ALGA)}

In this method, a sentiment lexicon is generated for the datasets in the training phase, and this lexicon is used for classification of tweets in the test phase. This lexicon contains sentiment words with a score assigned to them. This score is calculated and is in the range of [minneg, maxpos]. minneg and maxpos are the lowest and highest score a sentiment word can have.

\subsection{Sentiment Word Extraction}

All the words with frequencies higher than 2 in the training dataset are considered as sentiment words here; if a word appears once or twice, the algorithm could not find a proper value for it. All of the frequent words are considered because the tweets are very short, and each word in them can have an implication; also, because it is desirable to capture the meanings of words that existing lexicons do not usually cover.

\subsection{The Optimization Problem}

The objective of this work is to find a lexicon that minimizes the classification error. Here a feature is defined, named ALGA, to find the lexicon. Suppose that there are $p$ datasets and $q$ lexicons. For each tweet (record) $T_{i}$ in dataset $D_{m}$, using lexicon $L_{k}$, ALGA is calculated as the sum of scores for each word of the tweet based on the lexicon. It is defined as follows:

$$
\operatorname{ALGA}\left(D_{m}, T_{i}, L_{k}\right)=\sum_{w_{j} \in T_{i}} v_{k}\left(w_{j}\right)
$$


In which $w_{j}$ represents the sentiment words present in $T_{i}$, and $v_{k}\left(w_{j}\right)$ is the sentiment score of $w_{j}$ in $L_{k}$. When the ALGA feature is positive for a record, the algorithm predicts the tweet as positive and when it is not positive, the tweet is predicted as negative. Therefore, the predicted class is defined as follows:

$$
\operatorname{Predicted\_ Class}\left(D_{m}, T_{i}, L_{k}\right)=\left\{\begin{array}{l}
\text { Positive, if } A L G A\left(T_{i}, L_{k}\right)>0 \\
\text { Negative, if } \operatorname{ALGA}\left(T_{i}, L_{k}\right) \leq 0
\end{array}(2)\right.
$$

Using the ALGA feature, lexicons can be more or less accurate in predicting classes of tweets. The accuracy of a lexicon over a dataset, $\operatorname{Accuracy}\left(D_{m}, L_{k}\right)$, is defined as the ratio of number of correct predictions of tweets in $D_{m}$ using $L_{k}$ to the number of tweets in $D_{m}$ :

$$
\operatorname{Accuracy}\left(D_{m}, L_{k}\right)=\frac{\text { Number of Correct Predictions of Tweets in } D_{m} u \operatorname{sing} L_{k}}{\text { Number of Tweets in } D_{m}}(3)
$$

The optimization problem is defined in its general form as follows: For a given dataset, find lexicon $L_{\text {best }}$ that maximizes the accuracy. In the formal form:

$$
\text { for } D_{m}, L_{\text {best }}=\underset{L_{k}}{\operatorname{argmax}} \operatorname{Accuracy}\left(D_{m}, L_{k}\right)
$$

\subsection{Preprocessing}

The sentiment words in this work are all of the words with a frequency of higher than 2 in the training dataset. Emoticons are also considered as sentiment words. Also, the stop words are present. Some stop words may have sentimental implications, and some may not. In the learning phase, those stop words that should have been omitted may get a score near 0 , and hence do not have an impact in the classification phase. All the punctuation marks are omitted, and all the words are turned into lower case.

\subsection{Genetic Algorithm}

For finding a lexicon that maximizes the accuracy, a genetic algorithm is incorporated. A genetic algorithm runs on a population of chromosomes (here, chromosomes are arrays), and gradually 
makes the population better in terms of fitness. In each iteration of the algorithm, two chromosomes are selected for crossover, and they produce two children that can have mutations. After this phase, they can replace chromosomes in the population. The chromosomes are compared by their fitness values, which are calculated from a fitness function. This process is repeated until certain convergence criteria on the fitness values is met, or simply after a certain number of iterations. Since it is desirable to find a lexicon with maximum accuracy, each chromosome in the population represents a sentiment lexicon. Their fitness value shows how they perform in classifying the tweets.

Each element of a chromosome is designated for a word, and its value shows the sentiment score of that word. A chromosome representation is shown in Table 1. The chromosomes are initialized randomly.

Table 1. An initialization of chromosomes in the genetic algorithm

\begin{tabular}{|c|c|c|c|c|}
\hline & good & was & loveable & water \\
\hline Chromosome 1 & +8 & -6 & +7 & -2 \\
\hline Chromosome 2 & +1 & 0 & +10 & -1 \\
\hline Chromosome 3 & -9 & -1 & +1 & +8 \\
\hline
\end{tabular}

For calculating the fitness value of the chromosome number $k$ on a given dataset, the ALGA feature is used and the chromosome number $k$ is considered as the Lexicon $k\left(L_{k}\right)$. The accuracy measure (equation 3) can be used as the fitness function, but a fitness function is desirable that can better guide the algorithm through the iterations. The first suggestion is using the ALGA feature as the fitness function. For example, consider two chromosomes (lexicons) and a dataset consisting of one record (tweet) which is positive. Using the first chromosome, the ALGA feature value is -30 for that record. Using the second chromosome, the ALGA feature value is -60 . Here, both chromosomes classify the record incorrectly, but the first chromosome should have a better fitness value, since its error is lower. Hence, the algorithm gradually lessens errors by improving fitness values, and guides chromosomes towards better classifications. However, here a more subtle evaluation of 
fitness is used. The aim is not to put the algorithm in pressure for reducing ALGA errors instead of improving accuracy. To this end, a reward and penalty function is defined, as follows:

$$
R\left(D_{m}, T_{i}, k\right)=\left\{\begin{array}{c}
1, \quad \text { if } A L G A\left(D_{m}, T_{i}, k\right) \text { correctly predicts the class of } T_{i} \\
-\frac{\left|A L G A\left(D_{m}, T_{i}, L_{k}\right)\right|}{\omega}, \text { if } A L G A\left(D_{m}, T_{i}, L_{k}\right) \text { incorrectly predicts the class of } T_{i}
\end{array}\right.
$$

$\omega$ is the penalty factor for misclassification of a record. $R\left(D_{m}, T_{i}, k\right)$ rewards for correct predictions, and penalizes wrong predictions proportional to how much they were wrong. If $\omega$ is set to a high number, then the reward value becomes more powerful. If $\omega$ is set to a low number, the penalty for misclassification gains power. The value of $\omega$ is set manually.

The number 1 is used for all of the correct predictions, because the main objective is to lower errors in wrong predictions, not to have high ALGA values in correct predictions. The fitness of the chromosome $k$ on the dataset $D_{m}$ is calculated as follows in equation (6):

$$
\text { Fitness }\left(D_{m}, k\right)=\sum_{i=1}^{i<n u m b e r} \text { of tweets } R\left(D_{m}, T_{i}, k\right)(6)
$$

The pseudo-code for fitness evaluation of a chromosome is presented in Algorithm 1.

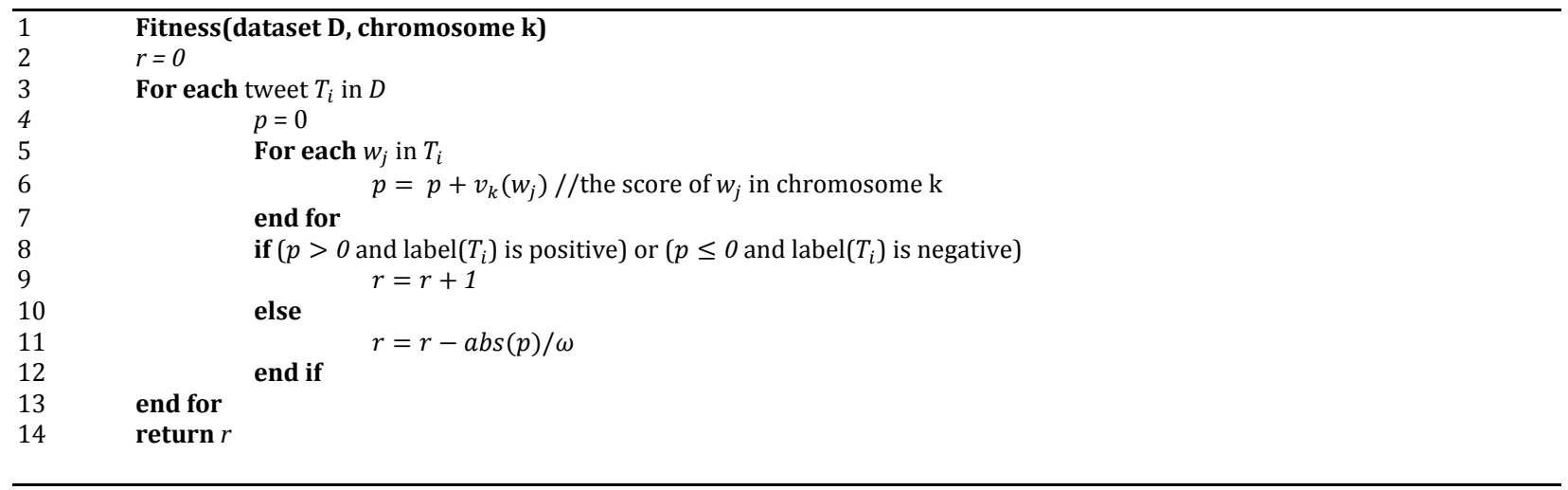

Algorithm 1. Fitness function of chromosome number $k$ in dataset $D$

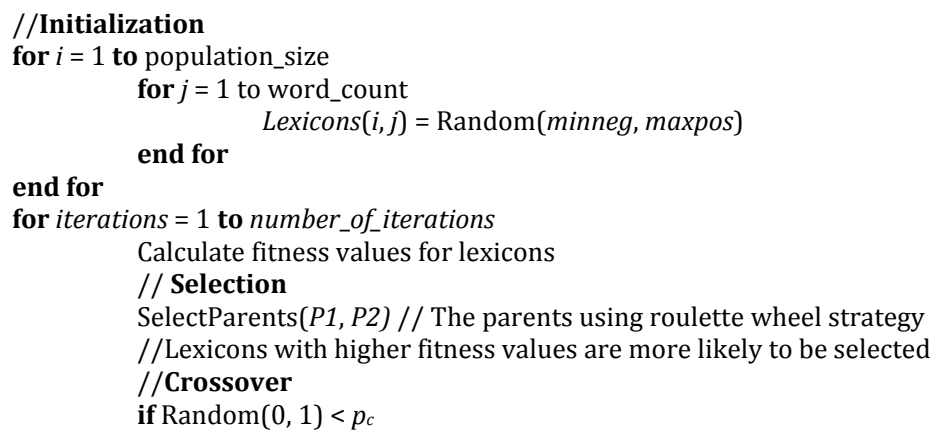




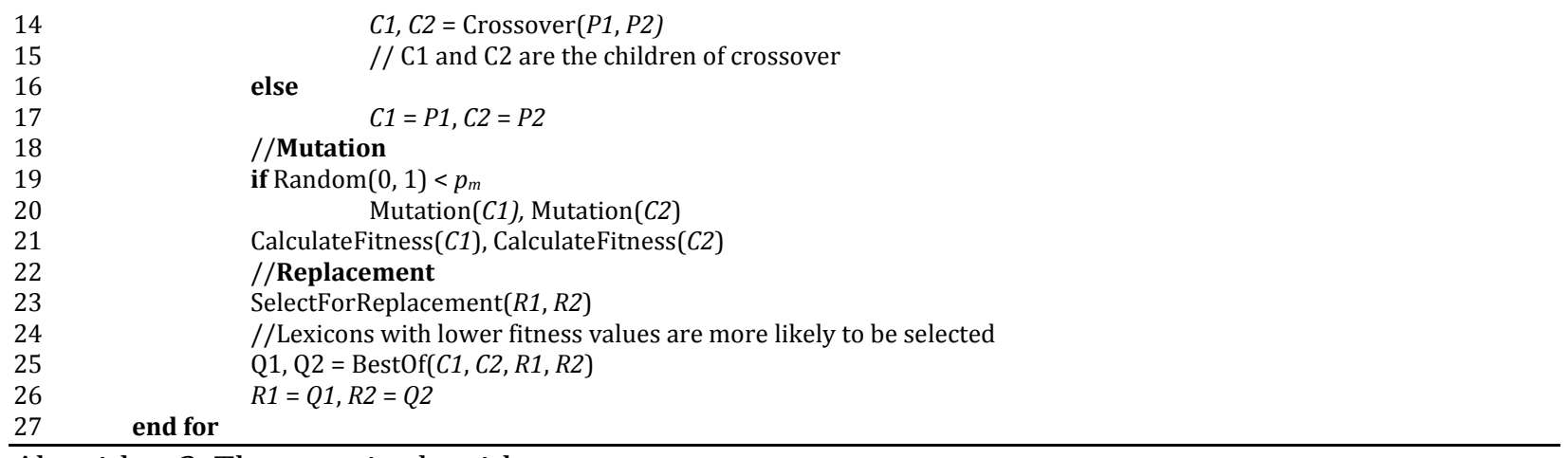

Algorithm 2. The genetic algorithm

The pseudocode for genetic algorithm is shown in algorithm 2, and runs as follows: first, all of the chromosomes in the population are initialized with random values between minneg and maxpos (line 4). Then, in the selection phase, two of the chromosomes are selected using the roulette wheel selection policy, in which the chromosomes with better fitness values have a higher chance of being selected (line 10). Afterwards, the crossover is applied with the probability of $p_{c}$ (line 13). The crossover method used here is uniform crossover, in which each gene in the children is randomly selected from either the first or the second parent. An example of uniform crossover is shown in figure 1.

\begin{tabular}{|c|c|c|c|c|c|c|c|c|c|}
\hline+6 & -1 & +8 & -7 & -3 & -5 & +4 & +3 & +4 & +1 \\
\hline+7 & +4 & -1 & +8 & -6 & -4 & +3 & +2 & +1 & -9 \\
\hline
\end{tabular}

Parent chromosomes

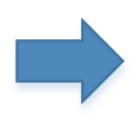

\begin{tabular}{|l|l|l|l|l|l|l|l|l|l|}
+7 & -1 & +8 & +8 & -3 & -4 & +3 & +3 & +1 & +1 \\
\hline
\end{tabular}

Child chromosomes

Figure 1: An example of uniform crossover

Then, with the probability of $p_{m}$, a mutation process is applied on the children (line 19). In this phase, each gene of child chromosomes is changed by a probability which will be described in section 4.2. Two of chromosomes in the population are selected for replacement with the roulettewheel strategy (line 23). In this strategy, the worse chromosomes in terms of fitness are more likely to be selected to be replaced. The two children and two selected chromosomes are compared and the best two are chosen (line 25), and they will have a place in the population (line 26). This process runs for 250,000 iterations so that the convergence can occur; in other words, after this 
number of iterations, the chromosomes are similar and continuing the algorithm is not necessary. In the experiments, in all datasets, after this number of iterations the chromosomes are almost identical and are converged. In the final round, the chromosome with the highest fitness value is selected as the final result and will be the lexicon to be used in the classification process.

\subsection{Polarity Classification}

In the test phase, for each record $T_{i}, \operatorname{ALGA}\left(D_{m}, T_{i}, L_{k_{\text {best }}}\right)$ is computed in which $k_{\text {best }}$ is the best chromosome in the final iteration in terms of fitness. If the score is greater than zero, the test record is assigned to the positive class; otherwise, it is assigned to the negative class. This is summarized in Algorithm 3.

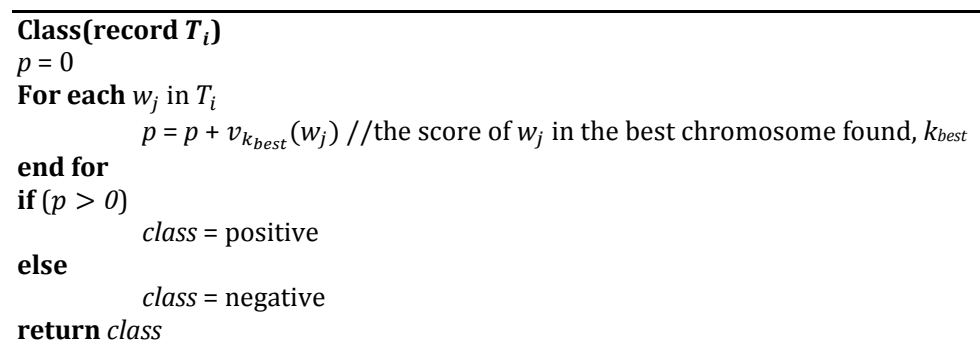

Figure 2 shows an example of classification using ALGA feature.

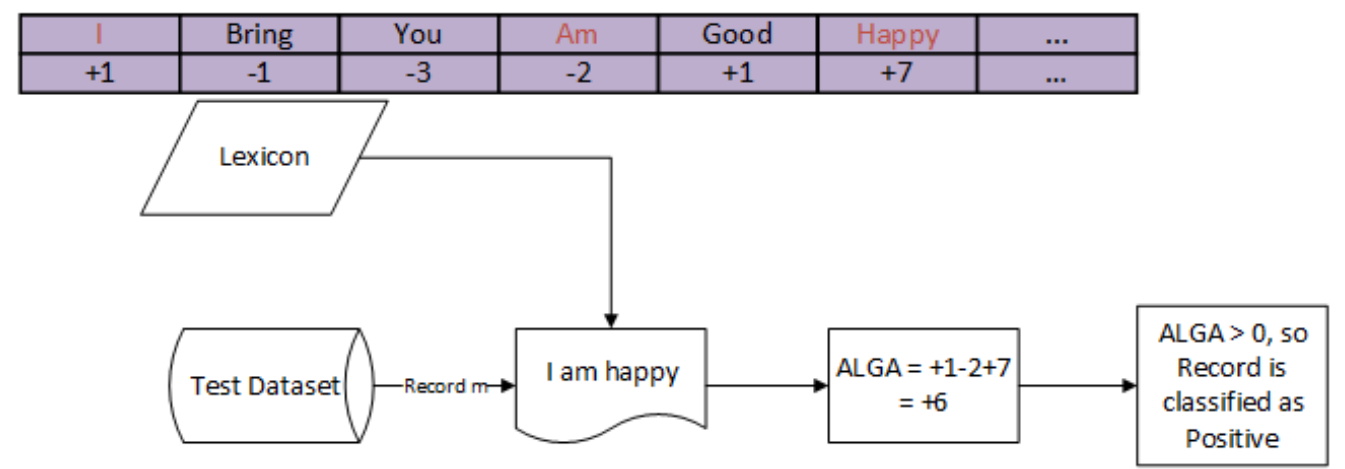

Figure 2. An example of classification of a tweet using a lexicon

\subsection{Additional Features}

After introducing the ALGA feature, additional features are used alongside ALGA. Two of them are meta-level features extracted using the Bing Liu lexicon [18], and the others are term frequencies of 
uni-, bi-, and trigrams in the text. The aforementioned two features from the lexicon are computed as follows:

BLP: Number of words present in the tweet that are positive in the Bing Liu lexicon BLN: Number of words present in the tweet that are negative in the Bing Liu lexicon For the classification purpose, SMO operator in WEKA is used, because of its high performance. The schematic model of this the proposed work is being presented in figure 3 . However, it should be noted that in the HCR dataset, the training and test datasets are previously known, and the procedure is repeated 100 times. The Train and Generate Lexicon using ALGA subprocess is done through a genetic algorithm, as it has been shown in figure 4 .

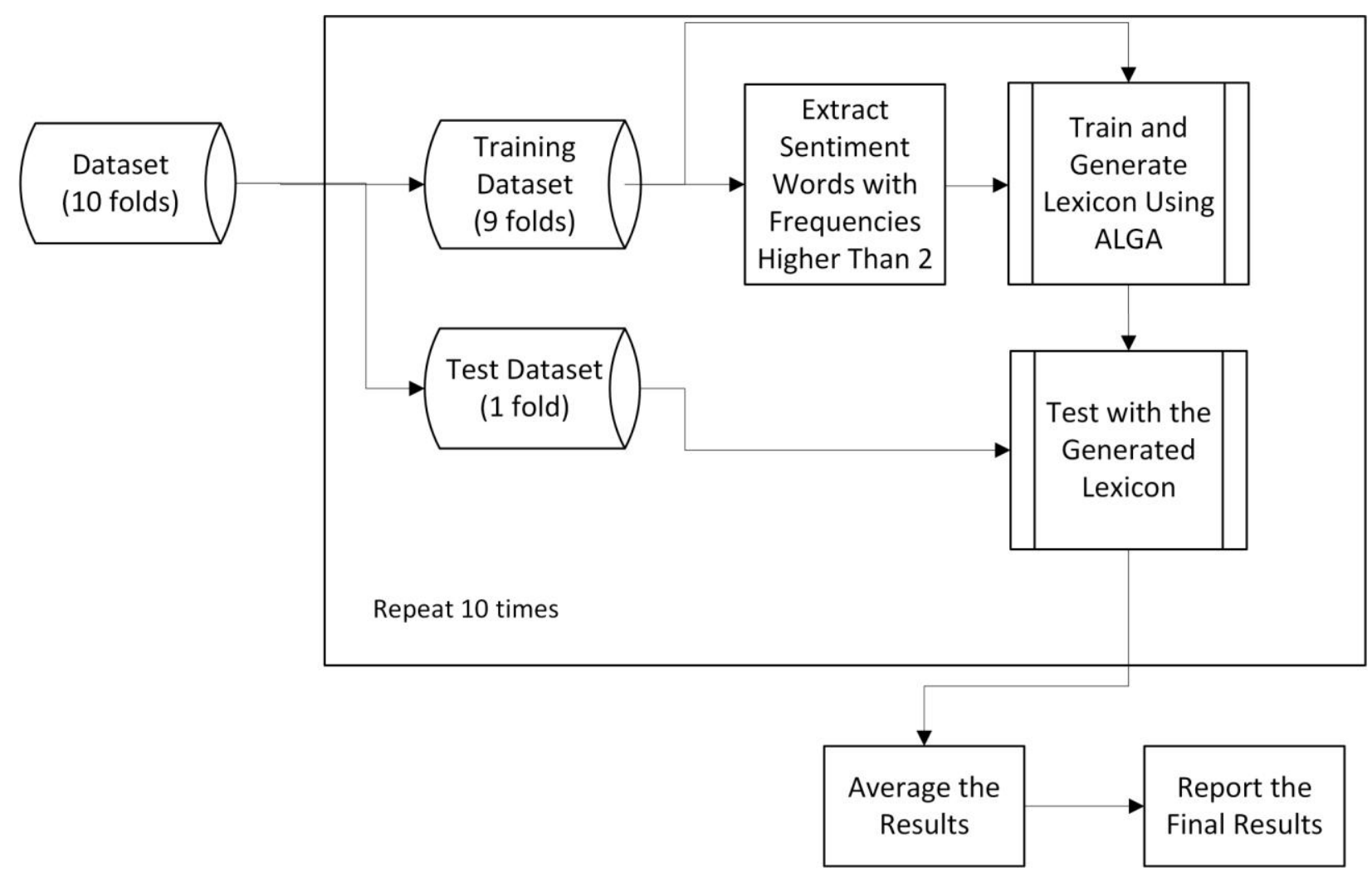

Figure 3. The schematic diagram of ALGA method

Figure 4 shows the genetic algorithm process for the training phase. The flowchart of fitness evaluation is shown in figure 5. In figure 5, a subprocess calculates the rewards and penalty values, and then the sum of errors is used as the fitness value of the chromosome. The calculate error 
subprocess is explained in figure 6. After training and generating the lexicon, the classification method based on ALGA should be used to classify the test dataset records into positive or negative classes. The flowchart for the classification process is shown in figure 7. When additional features are used, the summation value is reported as the ALGA feature, and is used alongside n-grams and the aforementioned BLP and BLN.

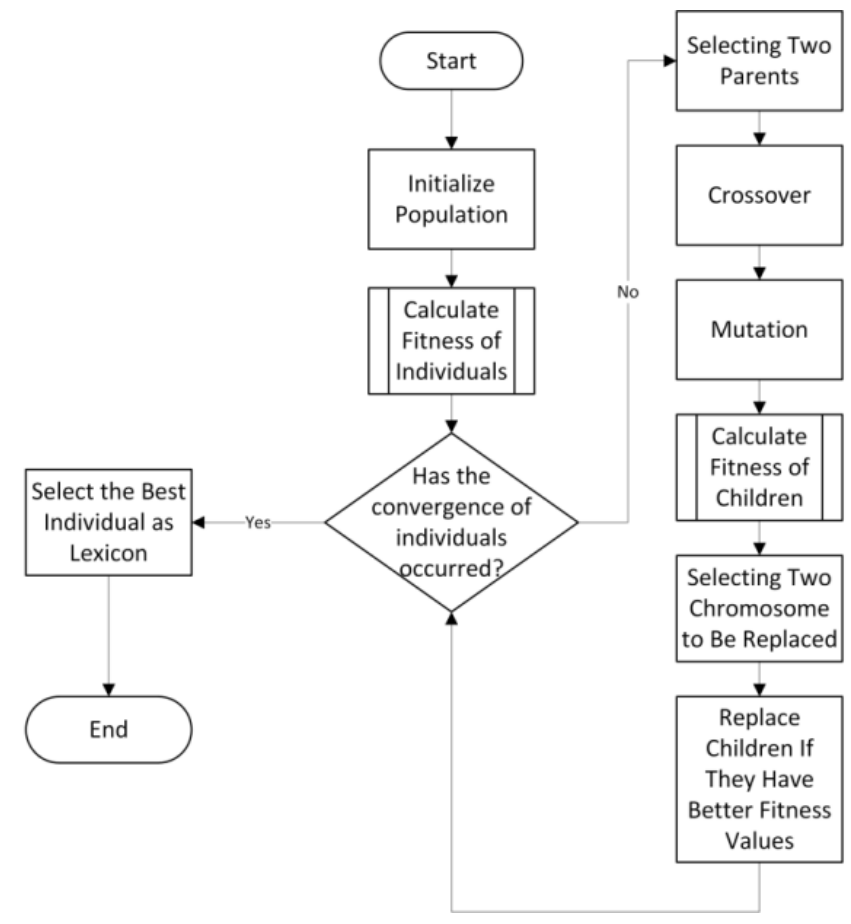

Figure 4. The training and generating lexicon process using ALGA flowchart 


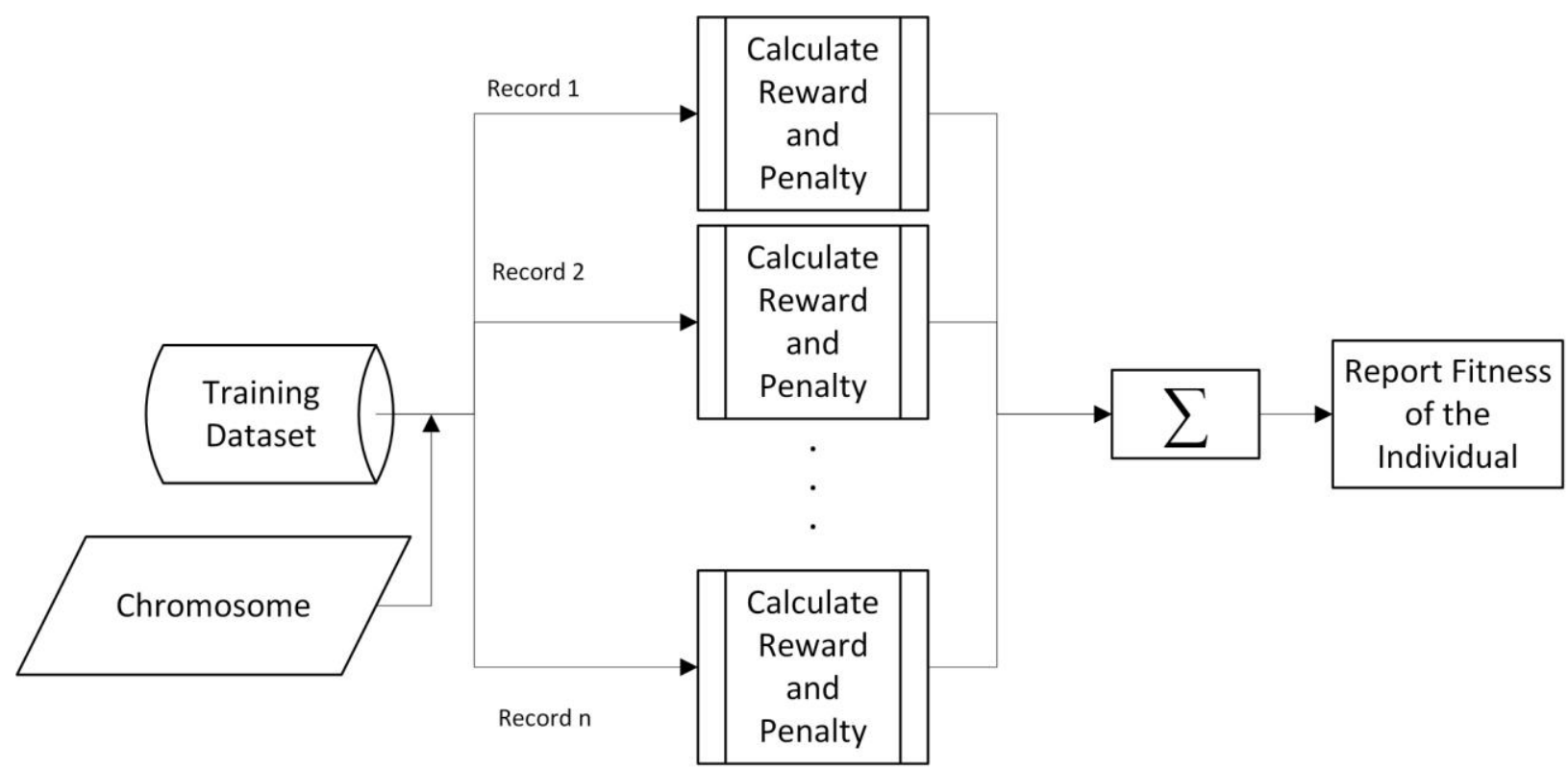

Figure 5. Calculating the fitness of a chromosome

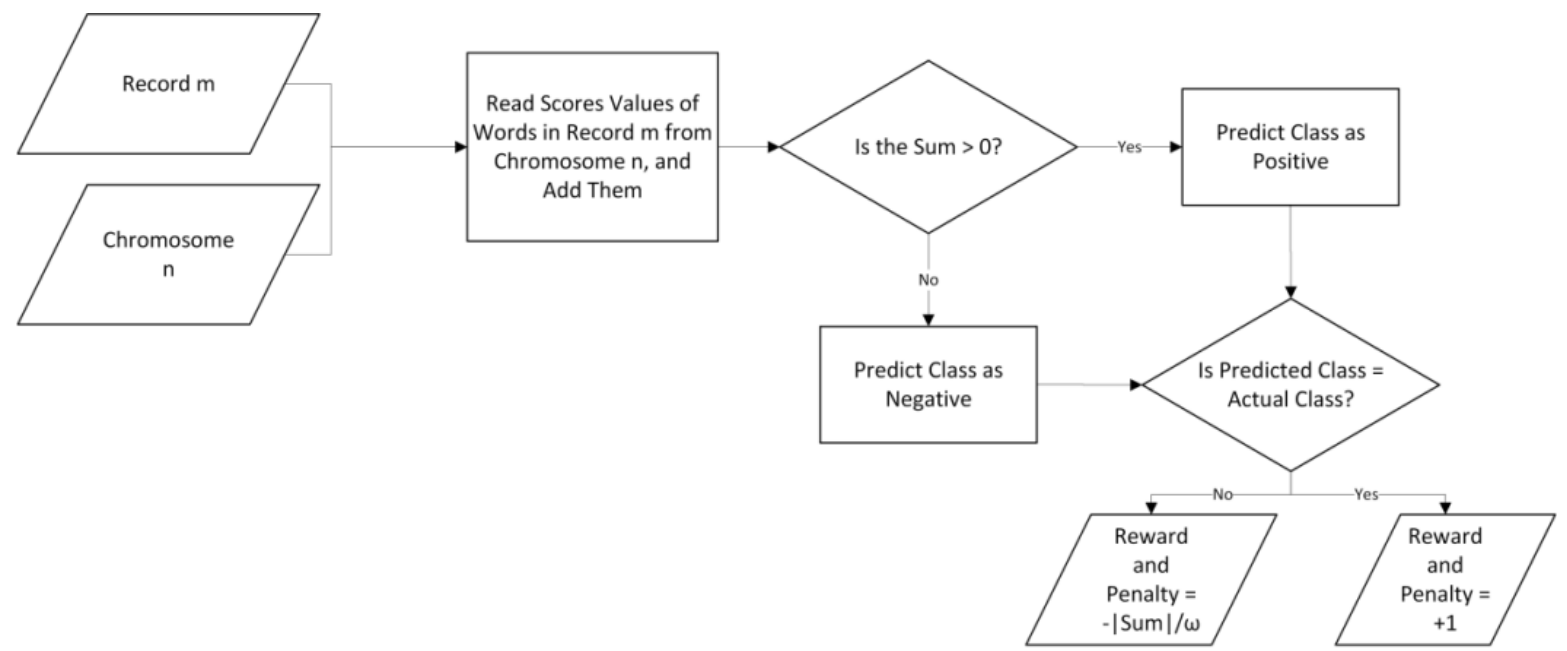

Figure 6. Calculating reward and penalty for record $m$ and chromosome $n$

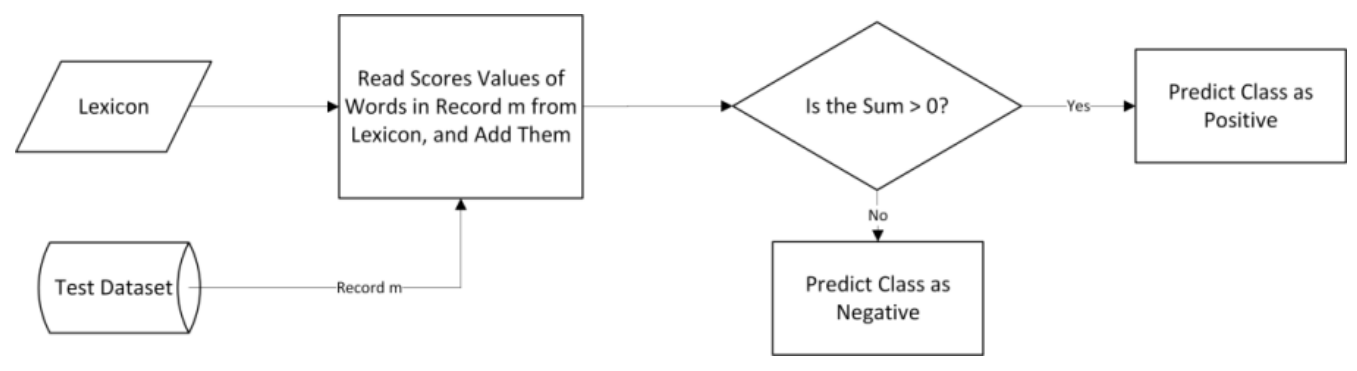

Figure 7. Predicting the class of record $m$ from test dataset using the generated lexicon 


\section{Experimental Evaluation}

In this section, the experimental evaluation of the proposed method is provided. The datasets and parameters are introduced, and the results of running the proposed method on the datasets are reported.

\subsection{Datasets}

ALGA was run on six different datasets made from tweets of users in Twitter [57]. These datasets are named Sanders, OMD, Strict OMD, HCR, SemEval and Stanford datasets.

\subsubsection{Sanders - Twitter Sentiment Corpus}

This corpus contains 570 positive and 654 negative tweets, labelled manually. The dataset is collected by searching @apple, \#google, \#microsoft, and \#twitter on the twitter. It has neutral and irrelevant tweets as well.

\subsubsection{Obama-McCain Debate (OMD) and Strict OMD datasets}

The original Obama-McCain Debate (OMD) dataset contains 3238 tweets that were posted during the presidential debate between Barrack Obama and John McCain in September 2008 [58]. Tweets are classified as positive, negative, mixed, or others by some voters. Since the votes may conflict, only the tweets with a majority vote are considered. In this version, 1906 tweets are extracted of which 1196 are negative and 710 are positive. Another version of this dataset is the Strict Obama McCain Debate dataset (SOMD), which consists of only the tweets where the votes are unanimous. SOMD has 347 positive and 569 negative tweets. 


\subsubsection{Healthcare Reform (HCR)}

The tweets of this dataset are collected using the hashtag "\#hcr" in March 2010 [59]. In this paper, only the tweets labelled as negative or positive are considered. This dataset consists of 621 tweets, from which 215 are positive and 406 are negative for the training phase, and 665 tweets, of which 154 are positive and 511 are negative for the test phase.

\subsubsection{SemEval Dataset}

The SemEval-2013 dataset [60] was built for Twitter sentiment analysis task (task 2) in the Semantic Evaluation of Systems challenge (SemEval-2013). 5098 tweets were retrieved for this dataset, of which 1458 tweets are negative and 3640 tweets are positive.

\subsubsection{STS Dataset}

The Stanford Twitter dataset was presented by Go et al. [20] and consists of a training dataset containing 1.6 million tweets that were labelled automatically using emoticons, and a test dataset (STS-Test) which is manually labelled. Like several previous studies, such as [57], this test dataset is used as the main dataset and the training dataset is discarded because its automatic labeling procedure is not sufficiently accurate. STS-Test contains 177 negative and 182 positive tweets. The results for the STS dataset are compared with both papers that use STS-Test for training and testing (in a k-fold cross-validation setting) and papers that train a model on the STS-Train dataset and test it on the STS-Test dataset. Table 2 shows a summary of these datasets.

Table 2. Negative, positive and total umber of tweets in the six datasets, which will be used for

\begin{tabular}{|c|c|c|c|c|c|c|}
\hline & Sanders & OMD & SOMD & HCR & SemEval & STS-Test \\
\hline Negative & 654 & 1196 & 347 & 369 & 1458 & 177 \\
\hline Positive & 570 & 710 & 569 & 917 & 3640 & 182 \\
\hline Total & 1224 & 1906 & 916 & 1286 & 5098 & 359 \\
\hline
\end{tabular}




\subsection{Experimental Setup}

A program was written in $\mathrm{C} \#$ to implement the genetic algorithm. The 10 -fold cross validation mechanism was used for the algorithm whenever the training and test datasets were not separated beforehand and the algorithm was ran 10 times on each of 10 folds, and the algorithm was ran on the HCR dataset 100 times and reported the average values. The sentiment scores for each sentiment word reported in this paper are achieved by running the algorithm on the whole dataset, instead of splitting it into training and test datasets. The number of population was set to 2000 . The minneg and minpos are set to -10 and +10 , respectively. The crossover is uniform, with crossover rate of 0.8 . The mutation rate was set at 0.05 , and in the mutation phase, each sentiment word value in the chromosome is changed randomly by a probability of 0.02 . The algorithm runs for 250,000 iterations to be considered converged. These parameters are set experimentally. In order to show the sensitivity of the proposed method to parameters, the method was run on two datasets with different parameters. A small dataset, STS, and a large dataset, Sanders were chosen to run the tests. The results for different parameter settings have been shown in Table 3. This table shows the best values for crossover and mutation rates.

Table 3: Accuracy and F-measure for the STS and Sanders dataset with different parameter settings

\begin{tabular}{|c|c|c|c|c|c|c|}
\hline \multicolumn{3}{|c|}{ Parameters } & \multicolumn{2}{|c|}{ STS } & \multicolumn{2}{|c|}{ Sanders } \\
\hline$\omega$ & $p_{c}$ & $p_{m}$ & Accuracy & F1-Score & Accuracy & F1-Score \\
\hline 1 & 0.8 & 0.05 & 74.46 & 73.74 & 80.57 & 80.27 \\
\hline 30 & 0.8 & 0.05 & 78.49 & 78.21 & 79.03 & 78.65 \\
\hline 60 & 0.8 & 0.05 & 76.68 & 76.31 & 80.67 & 80.67 \\
\hline 90 & 0.8 & 0.05 & 75.28 & 74.84 & 79.05 & 79.07 \\
\hline 60 & 0.8 & 0.01 & 76.09 & 75.79 & 79.58 & 79.00 \\
\hline 60 & 0.8 & 0.02 & 75.90 & 75.30 & 80.41 & 80.17 \\
\hline 60 & 0.8 & 0.10 & 76.62 & 75.64 & 79.74 & 79.46 \\
\hline 60 & 0.7 & 0.05 & 78.51 & 77.82 & 79.25 & 77.70 \\
\hline 60 & 0.9 & 0.05 & 77.12 & 77.04 & 79.72 & 79.65 \\
\hline 60 & 1.0 & 0.05 & 75.03 & 74.26 & 78.73 & 78.61 \\
\hline
\end{tabular}

For crossover and mutation rate settings, different parameter values were examined. The Fmeasure values for different parameter values for these two datasets can be seen in figures 8 and 9. The algorithm was run 10 times for each setting and dataset, and the average results are reported. The results of running the algorithm with these parameter values on the other datasets follow the 
same pattern. The experiments show that the best results are yielded when crossover rate is between 0.7 and 0.9 , and mutation rate is between 0.05 and 0.10 . To make the ALGA method independent of parameter values as much as possible, the crossover and mutation rates for all of the datasets were set to 0.8 and 0.05 , respectively. With these parameters of ALGA, the results of the proposed method on datasets of different sizes outperform the state-of-the-art methods (as can be seen later in Table 4).



Figure 8. The f-measure values for different crossover and mutation rates on the Sanders dataset 


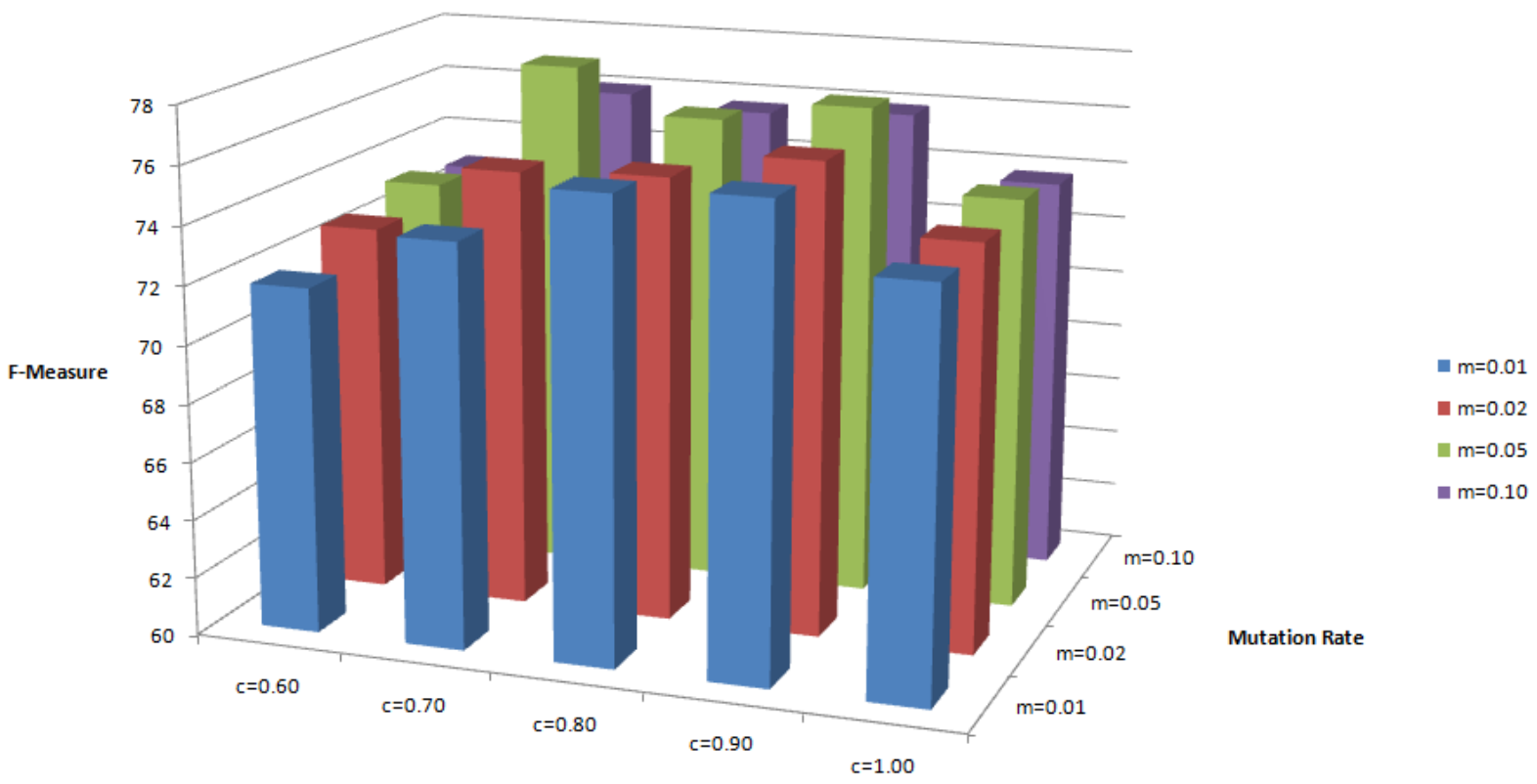

Crossover Rate

Figure 9. The f-measure values for different crossover and mutation rates on the STS dataset

On the number of iterations, 125,000 iterations for small datasets, such as the STS dataset are sufficient. The best chromosome does not change after this number of iterations in the majority of runs on this dataset. However, the best chromosome changes in the final iterations in larger datasets, such as Sanders and OMD. This leads to an overall slight improvement in accuracy and Fmeasure values in larger datasets. Due to this improvement, it was decided to keep the iterations number at 250,000, though the execution time increases. However, in all of the datasets, 50,000 iterations yield good enough results. The changes in accuracy in one run of ALGA on the OMD dataset over the iterations are shown in figure 10. 




Figure 10. The changes in accuracy in one run on the OMD dataset in iterations

In equation (5), a parameter is introduced which is named $\omega$. The experiments reveal that the best results are gained with $\omega=60$.

\subsection{Results and Discussion}

The results of the proposed method are compared with the best results from previous methods, Bing Liu's lexicon and a random search approach. After that, the results are discussed. The comparison is shown in Table 4. In this table, the bold values show the best results. In the random search, a single chromosome based on the representation in the genetic algorithm is initialized with random values. For 250,000 iterations, a neighbor of chromosome is generated by changing a single cell in it to a random value. If the neighbor has a higher fitness (as calculated in ALGA), it replaces the original one.

The ALGA-SW value is running ALGA without considering stop-words. ALGA + 1, 2, 3-grams shows the results of classification using ALGA feature and n-gram features. ALGA + lex is the result of classification using ALGA feature alongside two meta-level features from the Bing Liu's lexicon. 
Table 4. Results of running ALGA on the datasets

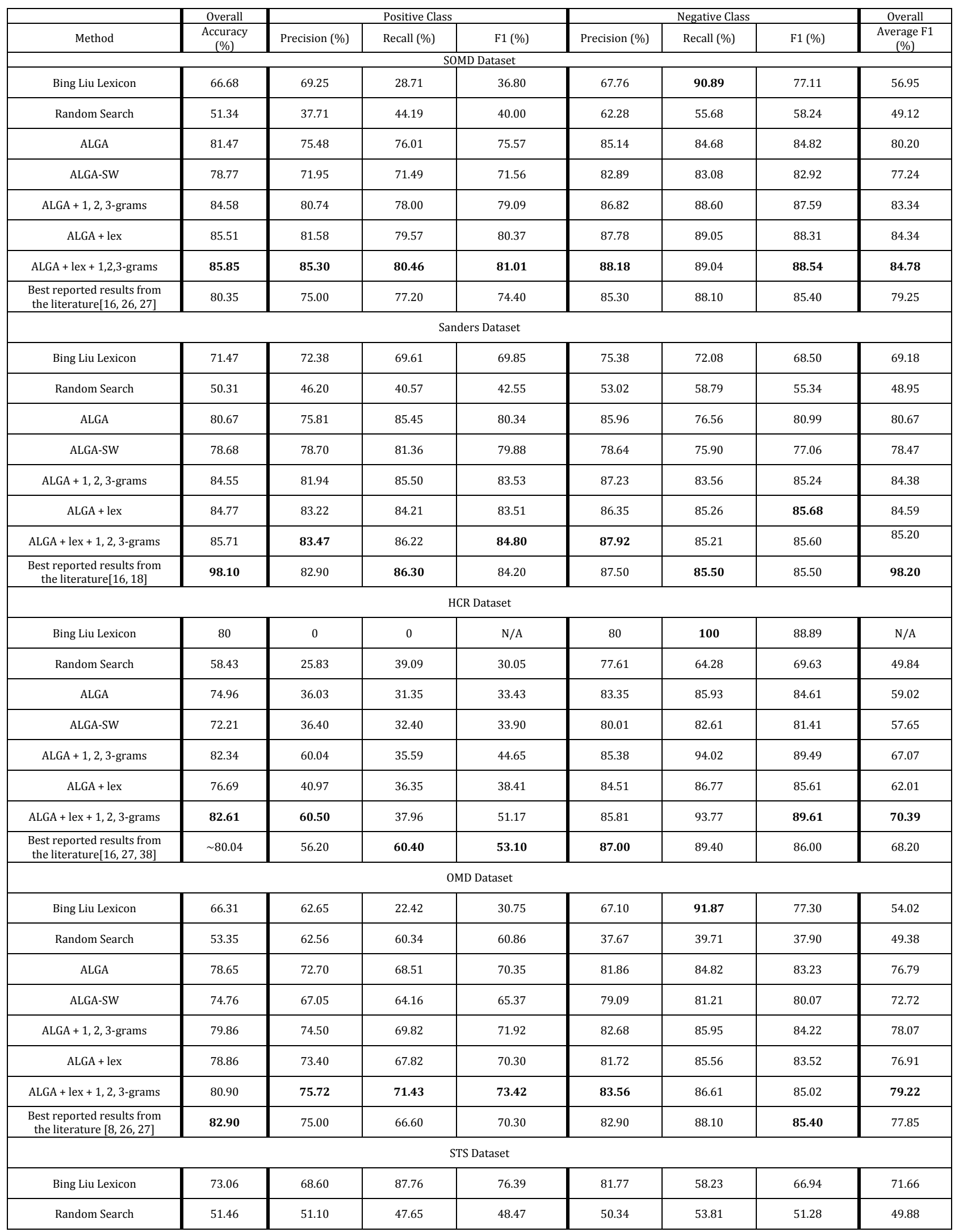




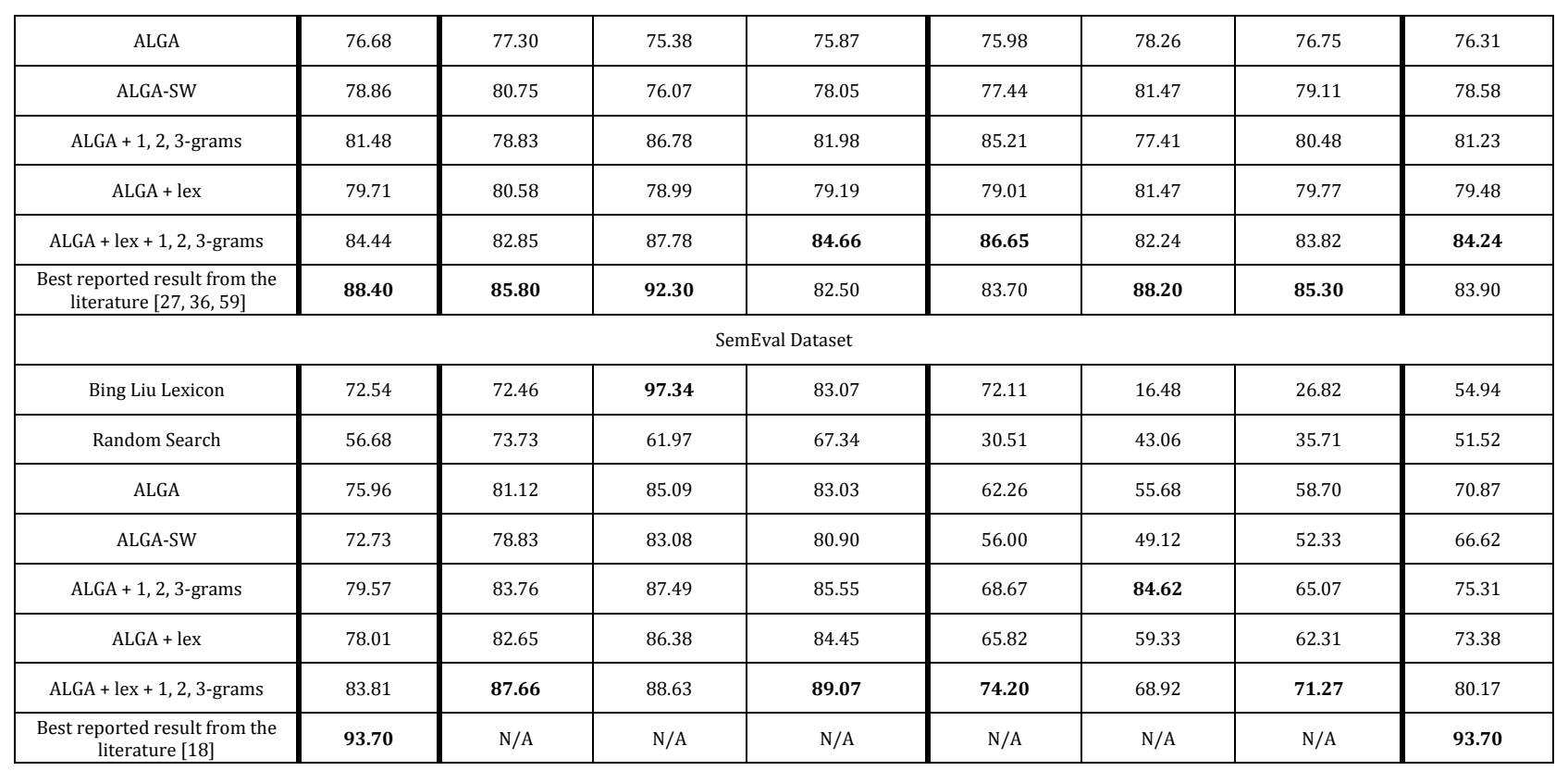

Since the genetic algorithm is run 10 times in each of the 10 folds, a confidence interval value should be reported. Table 5 shows the 0.95 confidence intervals for the proposed method on the six datasets.

Table 5. The 0.95 confidence interval for accuracy and F1-score values on the six datasets

\begin{tabular}{|l|c|c|c|c|c|c|}
\hline & SOMD & Sanders & HCR & OMD & STS-Test & SemEval \\
\hline Accuracy of ALGA & $81.47 \pm 2.13$ & $80.67 \pm 2.26$ & $74.96 \pm 1.71$ & $78.65 \pm 1.85$ & $76.68 \pm 2.52$ & $75.96 \pm 2.20$ \\
\hline F1-Score of ALGA & $80.20 \pm 2.13$ & $80.67 \pm 2.31$ & $59.02 \pm 1.69$ & $76.79 \pm 1.84$ & $76.31 \pm 2.55$ & $70.87 \pm 2.11$ \\
\hline Accuracy of ALGA + lex + n-grams & $85.85 \pm 2.08$ & $85.71 \pm 2.41$ & $82.61 \pm 1.70$ & $80.90 \pm 1.85$ & $84.44 \pm 2.52$ & $83.81 \pm 2.16$ \\
\hline F1-Score of ALGA + lex + n-grams & $84.78 \pm 2.09$ & $85.20 \pm 2.53$ & $70.39 \pm 2.75$ & $79.22 \pm 1.87$ & $84.24 \pm 2.51$ & $80.17 \pm 2.15$ \\
\hline
\end{tabular}

It should be noted using just the ALGA feature yields promising results comparing results from an ensemble of classifiers, such as [16], or use of extensive meta-level features generated using existing well-known lexicons [18]. Using just ALGA outperforms other methods in the Strict OMD dataset in most measures. The reason of better results in the Strict OMD dataset is its clarity about positivity and negativity, because it is consisted of records in the original OMD dataset in which all the votes are unanimous. This cannot be said for other datasets, and there are records in them that seem ambiguous in polarity. The investigation of falsely classified records in ALGA shows that it could be said that to some extent, the problem is the tone of tweet. 
Adding n-grams features and meta-level features of Bing Liu lexicon improves the results and makes them better in many measures in the datasets. The reason is that the less frequent words in the datasets tend to have higher variance values in multiple runs, and they are more prone to having less proper values. Figure 11 shows the standard deviation values of sentiment scores of words in the 100 runs of the HCR dataset, versus their frequency values. The less frequent a word, the probability of having a high standard deviation in the various runs gets higher.

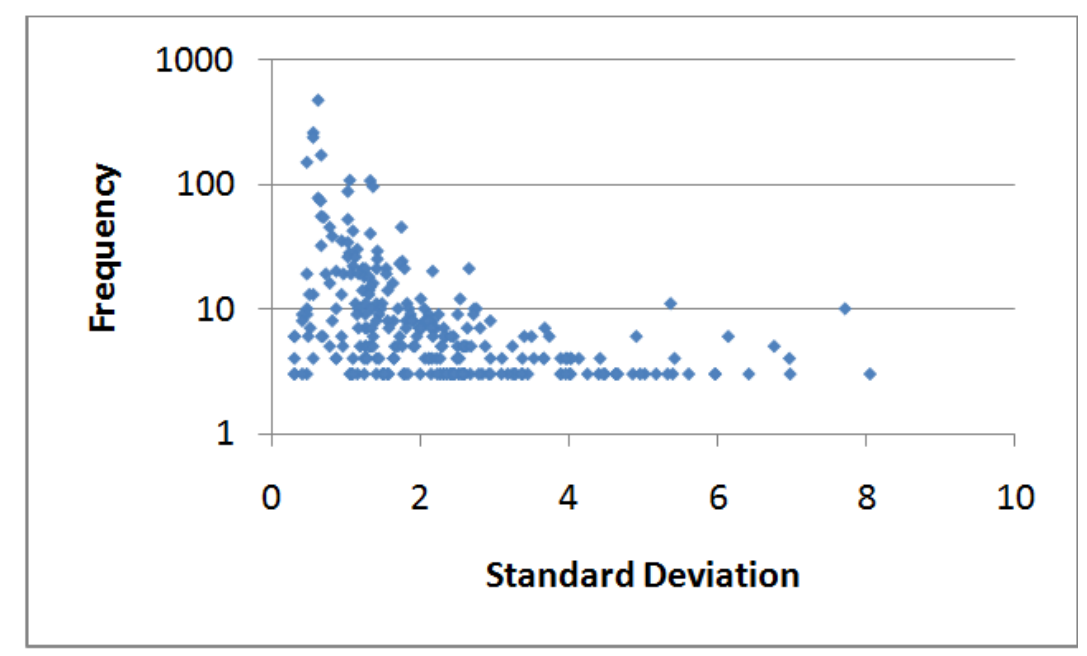

Figure 11: Standard Deviation vs. Frequency of Words in the HCR dataset

This problem can be solved by adding Bing Liu lexicon features or n-gram features. The BLP and BLN meta-level features have more correct values for these words. BLP and BLN, and ALGA tend to correct each other. However, ALGA has a higher granularity and can capture the sentiment scores of more frequent words better than Bing Liu's lexicon, and it shows itself in words with higher frequencies.

It can also be said for n-gram features. Here again, these features correct the decision when the ALGA feature is wrong, and vice versa. Using n-gram features leads to improvements in results. That can be related to the negation to some extent. ALGA only uses unigrams. Therefore, in a sentence like "It is not working", though "not" negates the sentiment of "working", the score of "not" 
is near -10 , and the score of "working" is close to +10 . Hence, they nullify each other and the decision is made based on the sentiment values of "it" and "is". However, when using n-grams, "not working" has a considerable negative weight. Bing Liu and n-gram features also show their impact on the unrepresented sentiment words because of low frequency in the text. They have a potential impact on classification, though scarcely used.

Though it seems that some words have higher impacts than the others, it was decided to include all of the words in the datasets with a frequency higher than 2. It was also decided to keep even those words that are conventionally omitted as stop-words. In Table 4, ALGA-SW shows the results of using ALGA without stop-words. Omitting the stop-words negatively affects the performance of the algorithm. It has been reported in [61] that it is better not to remove stop-words.

Figure 12 shows the overlapping of ALGA generated lexicons and the Bing Liu lexicon. The numbers show the number of words in each lexicon. The black number in the intersection shows the number of words on which both lexicons agree, and the white number represents their non-conformant words.

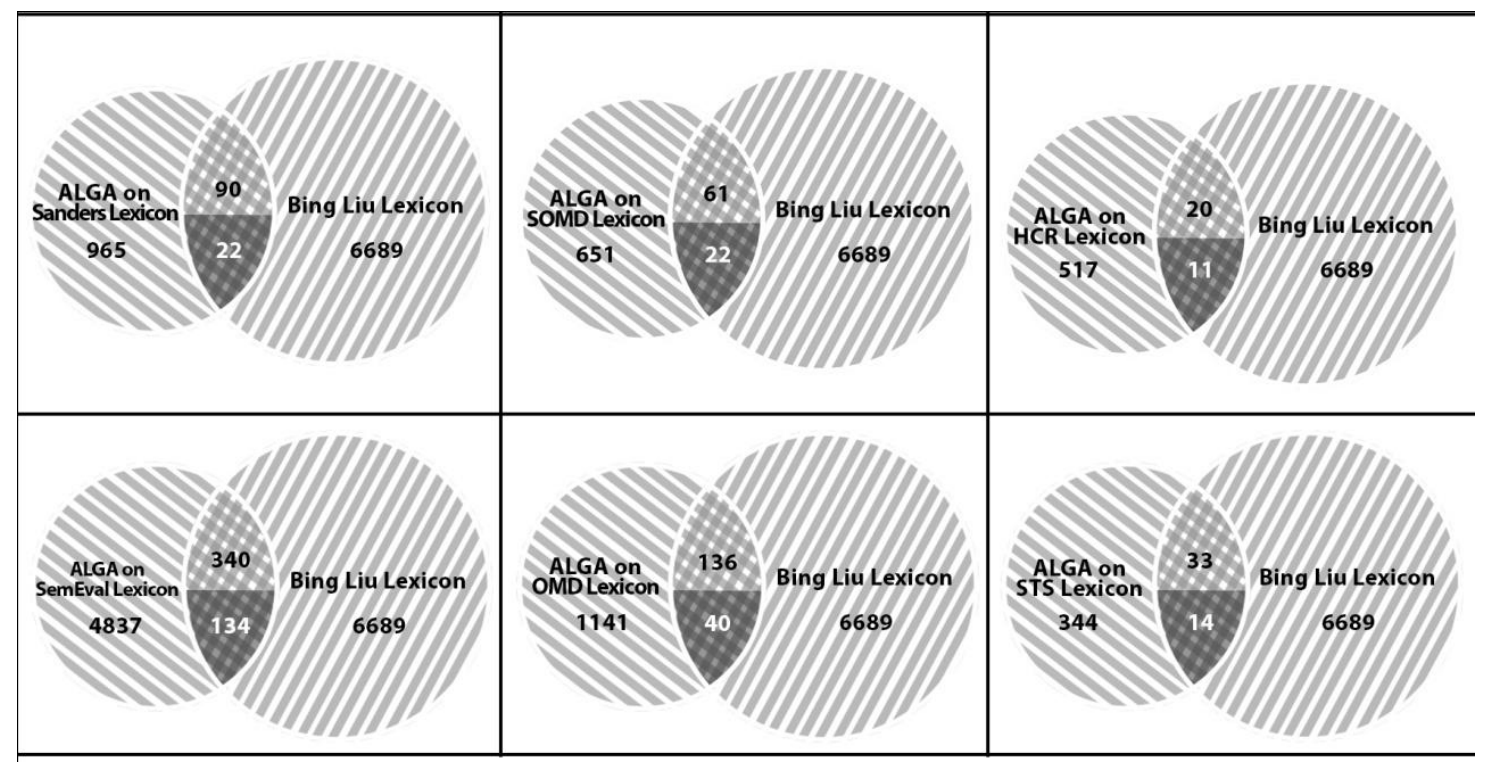

Figure 12. The venn diagram of words in lexicons, their overlap, and their concordance in polarity orientation (black number showing concordance, white number showing non-concordance) 
Table 6 shows the number of sentiment words for each dataset. These words are present in the sentiment lexicon that is constructed only for that dataset.

Table 6: The number of sentiment words for each dataset

\begin{tabular}{|l|c|c|c|c|c|c|}
\hline & ALGA on HCR & ALGA on SOMD & ALGA on Sanders & ALGA on OMD & ALGA on STS & ALGA on SemEval \\
\hline Length of Lexicon & 517 & 651 & 965 & 1141 & 344 & 4837 \\
\hline
\end{tabular}

Figure 13 show the distribution of sentiment word over classes. Each bar shows the number of sentiment words for each sentiment score (ranging from -10 to +10 ). The sentiment scores for each word are taken from running the algorithm on the whole dataset.

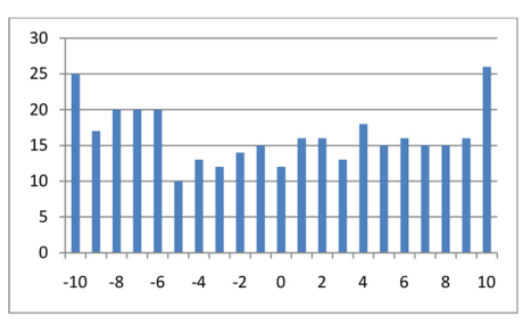

STS

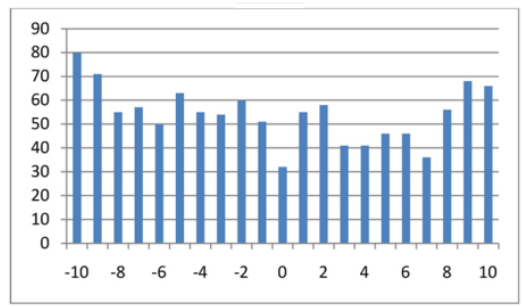

OMD

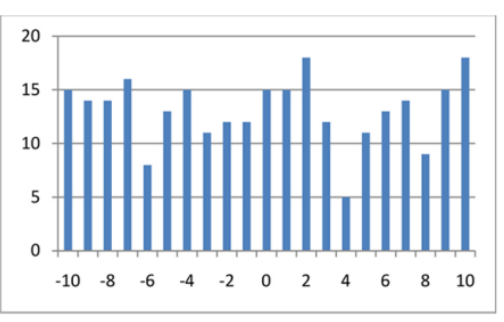

HCR



Sanders

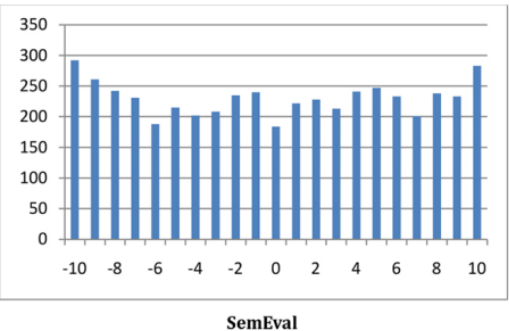

SemEval

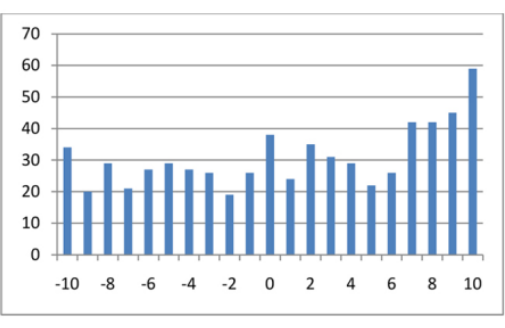

Strict OMD

Figure 13. Distribution of words in each score in datasets

Table 7: The most frequent words for each sentiment score in each lexicon

\begin{tabular}{|c|c|c|c|c|c|c|}
\hline Sentiment Score & HCR & Sanders & SOMD & STS & OMD & SemEval \\
\hline-10 & \#tcot & Not & -1 & So & -1 & Not \\
\hline-9 & About & When & Not & Time & Not & No \\
\hline-8 & What & All & McCain & $\mathrm{Me}$ & 1 & Even \\
\hline-7 & No & Of & $\mathrm{He}$ & This & 2 & If \\
\hline-6 & $\mathrm{He}$ & \#samsung & When & Go & $\mathrm{A}$ & How \\
\hline-5 & \#hcr & Shit & Is & And & McCain & They \\
\hline-4 & Will & Is & \#debate08 & On & \#Current & That \\
\hline-3 & \#tlot & Your & $\mathrm{A}$ & Back & $\mathrm{He}$ & $\mathrm{Me}$ \\
\hline-1 & The & To & You & Just & To & Have \\
\hline $\mathbf{0}$ & To & For & In & The & Is & Of \\
\hline 1 & In & And & That & I & \#tweetdebate & And \\
\hline 2 & That & The & I & It & $\mathrm{Be}$ & The \\
\hline 3 & Why & I & \#tweetdebate & To & On & For \\
\hline 4 & $\mathrm{We}$ & In & Just & As & Have & At \\
\hline 5 & Have & From & With & Will & I & You \\
\hline 6 & For & Sandwich & Have & My & Job & 2nd \\
\hline 8 & \#hhrs & \#google & Debate & Have & Tonight & Come \\
\hline
\end{tabular}




\begin{tabular}{|c|c|c|c|c|c|}
\hline $\mathbf{9}$ & Tell & Thanks & Obama & New & Obama \\
\hline $\mathbf{1 0}$ & Yes & Love & Good & Love & Good \\
\hline
\end{tabular}

Table 7 shows that some words that are often not included in the sentiment lexicons or are filtered as stop words can be decisive in sentiment classification. For example, the word "for" is considered a stop-word, but it has a rather high positive score in some datasets. The word "is" also has a negative sentiment score. Values for some stop-words in the proposed lexicons are presented in Table 8.

Table 8: Scores for some of the words in three datasets

\begin{tabular}{|c|c|c|c|c|c|c|}
\hline Word/Score & $\begin{array}{c}\text { ALGA on } \\
\text { HCR }\end{array}$ & $\begin{array}{c}\text { ALGA on } \\
\text { Sanders }\end{array}$ & $\begin{array}{c}\text { ALGA on } \\
\text { SOMD }\end{array}$ & $\begin{array}{c}\text { ALGA on } \\
\text { STS }\end{array}$ & $\begin{array}{c}\text { ALGA on } \\
\text { OMD }\end{array}$ & $\begin{array}{c}\text { ALGA on } \\
\text { SemEval }\end{array}$ \\
\hline About & -9 & 3 & -9 & -4 & -2 & 1 \\
\hline Anything & -9 & -4 & 5 & N/A & 4 & -1 \\
\hline Them & -7 & -7 & -7 & -1 & 2 & -1 \\
\hline Have & 5 & 2 & 6 & 8 & 4 & -1 \\
\hline For & 6 & 0 & 7 & 3 & 7 & 3 \\
\hline
\end{tabular}

It can be deducted that some stop words do have a sentimental value, although an implicit one. The proposed method can capture these sentimental values.

Table 9 shows the neutrality of each lexicon [18]. For the experiments, these sentiment values were considered as neutral scores: $-1,0$, and +1 .

Table 9: Neutrality of Lexicons [18]

\begin{tabular}{|c|c|}
\hline Lexicon & Neutrality \\
\hline AFINN & 0 \\
\hline Sentiment140 & 0.62 \\
\hline NRC-hashtag & 0.72 \\
\hline Bing Liu & 0 \\
\hline ALGA on HCR & 0.17 \\
\hline ALGA on Sanders & 0.14 \\
\hline ALGA on SOMD & 0.14 \\
\hline ALGA on STS & 0.12 \\
\hline ALGA on OMD & 0.12 \\
\hline ALGA on SemEval & 0.13 \\
\hline
\end{tabular}


Most of the words in Sentiment140 and NRC-hashtag are neutral. The lexicons created by ALGA have common words with these lexicons, but in the generated lexicons, most of these words are not neutral.

Figures 14 to 19 show the box plot for ALGA values for correct and wrong predictions. These boxplots show the $0^{\text {th }}, 25^{\text {th }}, 50^{\text {th }}, 75^{\text {th }}$ and $100^{\text {th }}$ percentiles of the ALGA value. As it is shown in these figures, when ALGA feature correctly predicts the class of a record, it tends to have a more positive (for positive class) or more negative (for negative class) value than when it wrongly predicts the class. It shows that when ALGA feature is wrong, it is close to predicting correctly.

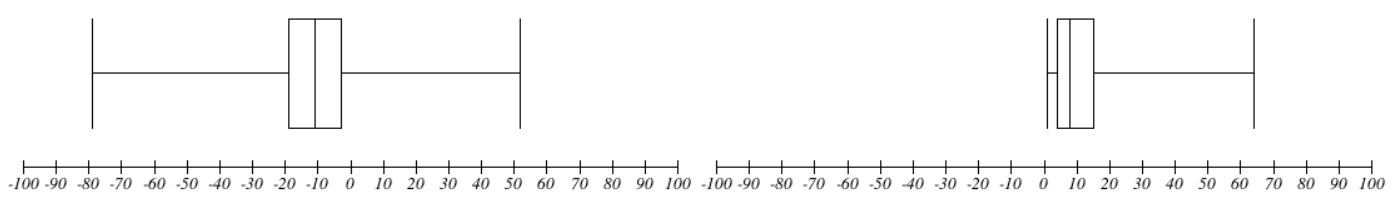

Figure 14: ALGA feature value for correct (left) and wrong (right) predictions of the HCR Dataset

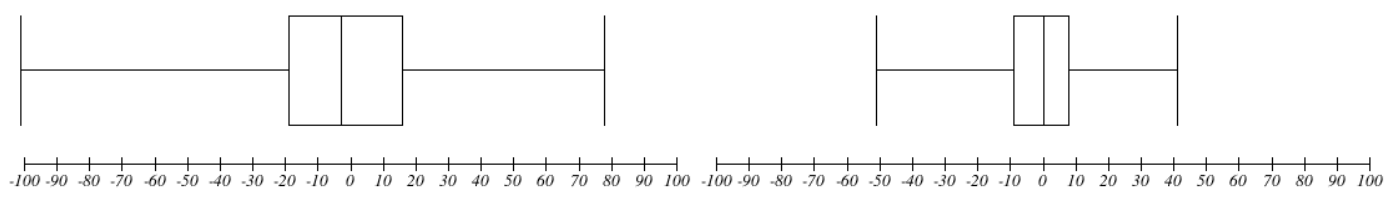

Figure 15: ALGA feature value for correct (left) and wrong (right) predictions of the Sanders Dataset

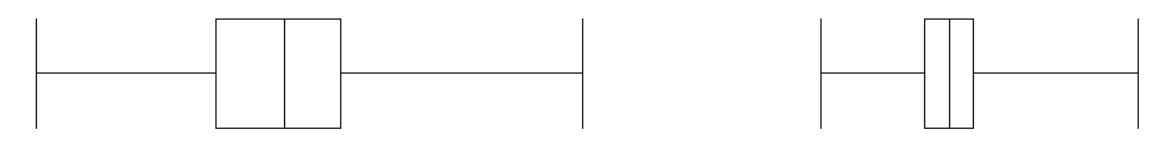

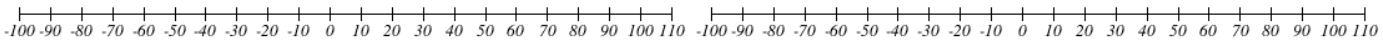

Figure 16: ALGA feature value for correct (left) and wrong (right) predictions of the SOMD Dataset
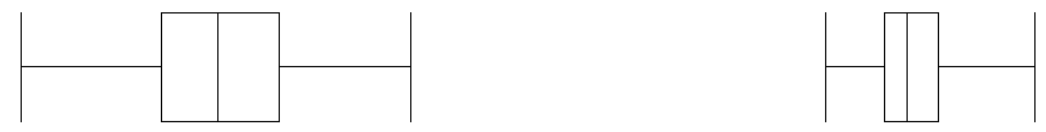
Figure 17: ALGA feature value for correct (left) and wrong (right) predictions of the STS dataset

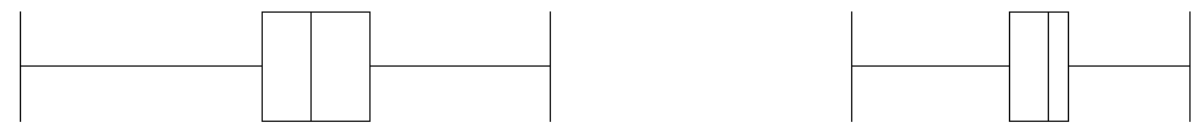

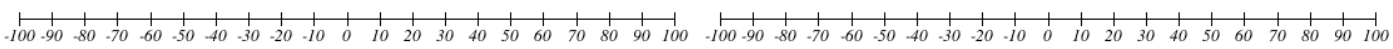

Figure 18: ALGA feature value for correct (left) and wrong (right) predictions of the OMD dataset

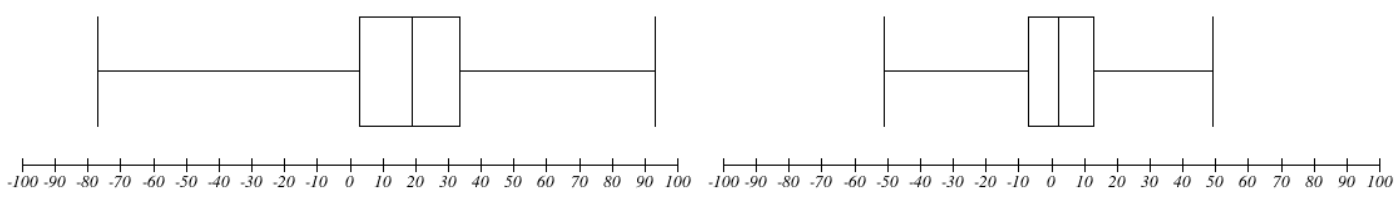

Figure 19: ALGA feature value for correct (left) and wrong (right) predictions of the SemEval dataset

\subsection{Statistical Test}

The Wilcoxon Signed-Ranks test is performed on the results, which is a statistical non-parametric test, for calculating how well the proposed method works comparing to other methods. The results can be seen in Tables 10 and 11. The paper [18] is a straightforward method with high accuracy and F-measure values. It was applied on only three of the six datasets, so the method was implemented on the remaining three datasets and the results are considered in Tables 10 and 11.

Table 10: Wilcoxon Signed-Ranks test on accuracy values

\begin{tabular}{|c|c|c|c|c|c|c|c|c|c|}
\hline Paper & Wins & Losses & Mean (ALGA) & Mean (Paper) & $\begin{array}{l}\text { Standard } \\
\text { Deviation (ALGA) }\end{array}$ & $\begin{array}{l}\text { Standard } \\
\text { Deviation (Paper) }\end{array}$ & $\mathrm{Z}$ & Asymp. Sig. (2-tailed) & Exact Sig. (2-tailed) \\
\hline [27] & 3 & 0 & 82.65 & 76.85 & 1.77 & 6.19 & -1.604 & 0.109 & 0.250 \\
\hline [59] & 2 & 1 & 82.65 & 74.20 & 1.77 & 9.37 & -1.069 & 0.285 & 1.000 \\
\hline$[32]$ & 1 & 1 & 81.75 & 79.05 & 1.21 & 4.88 & -0.447 & 0.655 & 1.000 \\
\hline [16] & 5 & 0 & 83.90 & 80.02 & 2.12 & 3.33 & -2.023 & 0.043 & 0.063 \\
\hline [31] & 4 & 0 & 83.41 & 79.70 & 2.10 & 3.22 & -1.826 & 0.068 & 0.125 \\
\hline [33] & 4 & 1 & 83.49 & 80.69 & 1.83 & 3.05 & -1.483 & 0.138 & 0.375 \\
\hline [37] & 2 & 0 & 81.75 & 68.09 & 1.21 & 1.56 & -1.342 & 0.180 & 0.500 \\
\hline$[56]$ & 2 & 0 & 81.75 & 76.35 & 1.21 & 1.20 & -1.342 & 0.180 & 0.500 \\
\hline$[34]$ & 2 & 0 & 85.07 & 83.41 & 0.90 & 0.61 & -1.342 & 0.180 & 0.500 \\
\hline [18] & 4 & 2 & 83.89 & 83.30 & 1.90 & 10.04 & -0.105 & 0.916 & 0.688 \\
\hline [28] & 2 & 0 & 85.07 & 80.45 & 0.90 & 0.50 & -1.342 & 0.180 & 0.500 \\
\hline [35] & 2 & 0 & 82.67 & 70.90 & 2.50 & 2.38 & -1.342 & 0.180 & 0.500 \\
\hline$[26]$ & 2 & 0 & 82.67 & 77.95 & 2.50 & 2.33 & -1.342 & 0.180 & 0.500 \\
\hline
\end{tabular}

Table 11: Wilcoxon Signed-Ranks test on F-measure values

\begin{tabular}{|l|l|l|l|l|l|l|l|l|l|}
\hline Paper & Wins & Losses & Mean (ALGA) & Mean (Paper) & $\begin{array}{l}\text { Standard } \\
\text { Deviation (ALGA) }\end{array}$ & $\begin{array}{l}\text { Standard } \\
\text { Deviation (Paper) }\end{array}$ & Z & Asymp. Sig. (2-tailed) & Exact Sig. (2-tailed) \\
\hline$[27]$ & 3 & 0 & 77.95 & 75.95 & 7.01 & 9.05 & -1.604 & 0.109 & 0.250 \\
\hline
\end{tabular}




\begin{tabular}{|l|l|l|l|l|l|l|l|l|l|}
\hline$[16]$ & 5 & 0 & 80.77 & 76.70 & 6.29 & 8.11 & -2.023 & 0.043 \\
\hline$[31]$ & 4 & 0 & 79.76 & 74.08 & 6.78 & 10.36 & -1.826 & 0.068 \\
\hline$[33]$ & 4 & 1 & 79.84 & 77.80 & 5.87 & 5.53 & -1.214 & 0.225 \\
\hline$[37]$ & 2 & 0 & 74.80 & 62.11 & 6.24 & 5.54 & -1.342 & 0.180 \\
\hline$[18]$ & 4 & 2 & 80.67 & 80.24 & 5.62 & 13.86 & -0.125 & 0.105 & 0.917 \\
\hline$[28]$ & 2 & 0 & 84.72 & 80.90 & 0.68 & 0.14 & -1.342 & 0.180 \\
\hline
\end{tabular}

\subsection{Delicate Meanings of Words}

ALGA is capable of capturing delicate meanings in Twitter culture. There are words that may seem positive, but have negative implications and vice versa. The proposed method gives a score of -10 to +10 , and if the score of a word is +8 , it can be said that it is more positive than a word with a score of +4 . For instance, the word "dear" in the generated lexicons gets a very negative score, though it is a positive word in AFINN, SenticNet, NRC-Emotion, and SentiWordNet. In Twitter, "dear" is mostly used in ironic tweets, such as "Dear @apple My new Air is now a notbook [sic] since your update killed \#wifi \#bug \#destroying \#productivity". The word "genius" seems positive, but when appearing in a sentence containing the word "Apple", it may refer to the "genius bar", thus making it an objective word, or it may be used as an irony. Another example is "Sorry". It appears more in positive tweets such as "Sorry \#Apple \#Google and \#Samsung just made you look bad. \#Android is king", and gets a mildly positive score. On the other hand, the word "Wow" gets mildly positive, or in some cases negative scores. It is because of its appearance in negative tweets such as “@APPLE Wow @MOTOROLA Just crushed your dreams....”. It is positive in Bing Liu's lexicon, and its score in AFINN is 4 out of 5.

\subsection{Limitations}

The main drawbacks of ALGA are as follows: (i) the lexicon creation phase is time-consuming, especially when the dataset is large (such as the SemEval dataset); however, after creating the lexicons, they can be used very fast to classify records; (ii) the sentiment scores of words in different domains are different and a comprehensive lexicon that works on domains is difficult to build using ALGA. 


\section{Conclusion and Future Work}

In this section, the paper is concluded, the results are assessed, and the implications of the proposed method and future works are discussed.

In this paper, it was demonstrated that using genetic algorithms in the search of optimal lexicons can lead to promising results. It was shown that using adaptive lexicons can lead to a better understanding of sentiment words. It was demonstrated that all of the words can be considered as sentiment words. ALGA had better accuracy results than state-of-the-art methods on two datasets, and better F-measure results in four datasets. The proposed method outperformed state-of-the-art methods such as [16], [31] and [34] in all of the datasets in terms of accuracy and F-measure. In Table 10, the accuracy results of the proposed algorithm were compared with 13 other methods. In this comparison, it was shown that the standard deviation of accuracy of ALGA on different datasets is lesser than 8 methods, and is slightly more than 5 methods. These 5 methods were applied on only two datasets. It shows that ALGA results usually vary less than other methods on different datasets.

It was shown that the F-measure of running ALGA is 5.53 percentage points better than the best reported results on the SOMD dataset, 2.19 on the HCR dataset, 1.37 on the OMD dataset and 0.34 on the STS dataset.

From the business intelligence point of view, it is important to get insight about the products and services companies provide. The companies can turn to social media to find out what users think about them using the lexicons created by ALGA. They can also analyze the tweets manually, with the knowledge that presence of what words are more important for classification, and what is the real meanings of words in Twitter using the ALGA created lexicons. A hotel manager can build a lexicon for the hotels and use it for sentiment analysis of their hotel. 
In ALGA, it is shown that even using a small dataset (STS-Test) is enough for achieving high accuracy and F-measure values and the number of tweets for training phase does not need to be high. Furthermore, it can be argued that if the value of ALGA feature is higher (lower) for a tweet, the tweet is more positive (negative). Using ALGA, the strength of opinions of tweets about a subject may be measured, and it will be explored in the future works. The lexicons can be updated online with active learning to adapt to the ever-changing culture of Twitter.

From a practical point of view, knowing the culture of Twitter and implications of words in specific domains makes users aware of words they use, for more effective transmission of their message.

This research has theoretical implications as well. It shows that a hybrid of lexicon-based and corpus-based methods can yield promising results, and genetic algorithm is useful for classifying opinionated text.

ALGA can be suitable for creating lexicons in languages that do not have one. This method also can capture the sentimental scores for same words in different cultures, domains, and websites.

In the future works, the modification of the proposed method for strength and emotion classification will be explored. Other evolutionary algorithms for the proposed problem will be examined as well. Changing the optimization problem will also be considered. Finally, it is planned to generate lexicons for other languages.

\section{Acknowledgements}

This work was supported by Iran National Science Foundation (INSF) (Grant No. 93036378).

\section{References}

[1] B. Liu, Sentiment analysis and opinion mining, Morgan \& Claypool Publishers, 2012.

[2] E. Fersini, E. Messina, F. A. Pozzi, Sentiment analysis: Bayesian Ensemble Learning, Decision Support Systems 68 (2014): 26-38. 
[3] R. Feldman, Techniques and applications for sentiment analysis, Communications of the ACM 56.4 (2013): 82-89.

[4] B. Liu, Sentiment analysis: A multi-faceted problem, IEEE Intelligent Systems 25.3 (2010): 76-

80.

[5] A. Ritter, S. Clark, O. Etzioni, Named entity recognition in tweets: an experimental study, Proceedings of the Conference on Empirical Methods in Natural Language Processing, EMNLP'11, Association for Computational Linguistics, Stroudsburg, PA, USA, (2011):1524-1534.

[6] P. D. Turney, Thumbs up or thumbs down?: semantic orientation applied to unsupervised classification of reviews, Proceedings of the 40th Annual Meeting on Association for Computational Linguistics, ACL 02, Association for Computational Linguistics, Stroudsburg, USA, (2002): 417-424. [7] K. Ravi, V. Ravi, A survey on opinion mining and sentiment analysis: tasks, approaches and applications, Knowledge-Based Systems 89 (2015): 14-46.

[8] B. He, C. Macdonald, J. He, I. Ounis, An effective statistical approach to blog post opinion retrieval, Proceedings of the 17th ACM Conference on Information and Knowledge Management, CIKM'08, ACM, New York, NY, USA, (2008): 1063-1072.

[9] A. Balahur, R. Steinberger, M. Kabadjov, V. Zavarella, E. van der Goot, M. Halkia, B. Pouliquen, J. Belyaeva, Sentiment analysis in the news, in Proceedings of the Seventh International Conference on Language Resources and Evaluation (LREC'10), ELRA, Valletta, Malta, 2010.

[10] F. H. Khan, U. Qamar, S. Bashir, SWIMS: Semi-supervised subjective feature weighting and intelligent model selection for sentiment analysis, Knowledge-Based Systems 100 (2016): 97-111. [11] M. S. Hajmohammadi, R. Ibrahim, A. Selamat, Cross-lingual sentiment classification using multiple source languages in multi-view semi-supervised learning, Engineering Applications of Artificial Intelligence 36 (2014): 195-203.

[12] Z. Cui, X. Shi, Y. Chen, Sentiment Analysis via Integrating Distributed Representations of Variable-length Word Sequence, Neurocomputing (2015): 126-132. 
[13] J. Weissbock, D. Inkpen, Combining textual pre-game reports and statistical data for predicting success in the national hockey league, Advances in Artificial Intelligence. Springer International Publishing (2014): 251-262.

[14] S. A. Bahrainian, M. Liwicki, A. Dengel, Fuzzy Subjective Sentiment Phrases: A Context Sensitive and Self-Maintaining Sentiment Lexicon, In Proceedings of the 2014 IEEE/WIC/ACM International Joint Conferences on Web Intelligence (WI) and Intelligent Agent Technologies (IAT)-Volume 01, (2014): 361-368.

[15] Y. Miura, S. Sakaki, K. Hattori, T. Ohkuma, TeamX: A sentiment analyzer with enhanced lexicon mapping and weighting scheme for unbalanced data. In Proceedings of the 8th International Workshop on Semantic Evaluation (2014): 628-632.

[16] N. F. F. da Silva, E. R. Hruschka, E. R. Hruschka, Tweet sentiment analysis with classifier ensembles, Decision Support Systems 66 (2014): 170-179.

[17] A. Hassan, A. Abbasi, D. Zeng, Twitter sentiment analysis: A bootstrap ensemble framework, 2013 International Conference on Social Computing (SocialCom), (2013): 357-364.

[18] F. Bravo-Marquez, M. Mendoza, B. Poblete, Meta-level sentiment models for big social data analysis, Knowledge-Based Systems 69 (2014): 86-99.

[19] P. Carvalho, L. Sarmento, M.J. Silva, E. de Oliveira, Clues for detecting irony in user-generated contents: oh...!! it's so easy;-), in Proceeding of the $1^{\text {st }}$ International CIKM Workshop on Topicsentiment Analysis for Mass Opinion, Hong Kong, China, (2009): 53-56.

[20] A. Go, R. Bhayani, L. Huang, Twitter sentiment classification using distant supervision, Technical report Stanford University, 2010.

[21] K. Liu, W. Li, M. Guo, Emoticon smoothed language models for Twitter sentiment analysis, in Proceedings of the 26th AAAI Conference on Artificial Intelligence, Toronto, Canada, 2012. 
[22] A. Agarwal, B. Xie, I. Vovsha, O. Rambow, R. Passonneau, Sentiment analysis of twitter data, in Proceedings of the Workshop on Languages in Social Media, LSM'11, Association for Computational Linguistics, Stroudsburg, PA, USA (2011): 30-38.

[23] J. Read, Using emoticons to reduce dependency in machine learning techniques for sentiment classification, Proceedings of the ACL Student Research Workshop, ACLstudent'05, Association for Computational Linguistics, Stroudsburg, PA, USA (2005): 43-48.

[24] L. Zhang, R. Ghosh, M. Dekhil, M. Hsu, B. Liu, Combining lexicon-based and learning based methods for twitter sentiment analysis, HP Laboratories, Technical Report HPL-2011 89, 2011. [25] S. Mohammad, S. Kiritchenko, X. Zhu, Nrc-Canada: building the state-of-the-art in sentiment analysis of tweets, in Proceedings of the Seventh International Workshop on Semantic Evaluation Exercises (SemEval-2013), Atlanta, Georgia, USA, 2013.

[26] X. Hu, L. Tang, J. Tang, H. Liu, Exploiting social relations for sentiment analysis in microblogging, in Proceedings of the Sixth ACM International Conference on Web Search and Data Mining, (2013): 537-546.

[27] H. Saif, Y. He, H. Alani, Semantic sentiment analysis of twitter, in Proceedings of the 11th International Conference on The Semantic Web, ISWC'12, Springer-Verlag, (2012): 508-524. [28] F. Bravo-Marquez, M. Mendoza, B. Poblete, Combining strengths, emotions and polarities for boosting twitter sentiment analysis, in Proceedings of the Second International Workshop on Issues of Sentiment Discovery and Opinion Mining, ACM, 2013.

[29] Y. Kaewpitakkun, K. Shirai, M. Mohd, Sentiment Lexicon Interpolation and Polarity Estimation of Objective and Out-Of-Vocabulary Words to Improve Sentiment Classification on Microblogging, in Proceedings of 28th Pacific Asia Conference on Language, Information and Computation, (2014): 204-213. 
[30] H. Saif, Y. He, M. Fernandez, H. Alani, Adapting Sentiment Lexicons Using Contextual Semantics for Sentiment Analysis of Twitter, The Semantic Web: ESWC 2014 Satellite Events, Springer International Publishing (2014): 54-63.

[31] L. F. S. Coletta, N. F. F. da Silva, E. R. Hruschka, E. R. Hruschka Jr, Combining Classification and Clustering for Tweet Sentiment Analysis, in Proceedings of 2014 Brazilian Conference on Intelligent Systems (BRACIS), 2014.

[32] T. J. Lu, Semi-supervised microblog sentiment analysis using social relation and text similarity, Big Data and Smart Computing (BigComp), 2015 International Conference on, IEEE, 2015.

[33] H. Saif, Y. He, M. Fernandez, H. Alani, Semantic patterns for sentiment analysis of Twitter, In The Semantic Web-ISWC (2014): 324-340, Springer International Publishing.

[34] C. Baecchi, T. Uricchio, M. Bertini, A. Del Bimbo, A multimodal feature learning approach for sentiment analysis of social network multimedia, Multimedia Tools and Applications (2015): 1-19. [35] X. Hu, J. Tang, H. Gao, H. Liu, Unsupervised sentiment analysis with emotional signals. In Proceedings of the 22nd international conference on World Wide Web (2013): 607-618.

[36] A. Bakliwal, P. Arora, S. Madhappan, N. Kapre, M. Singh, V. Varma, Mining sentiments from tweets. In Proceedings of the WASSA, 2012.

[37] H. Saif, M. Fernandez, Y. He, H. Alani. Senticircles for contextual and conceptual semantic sentiment analysis of twitter. In The Semantic Web: Trends and Challenges (2014): 83-98 [38] F. Wu, Y. Huang, Y. Song, Structured microblog sentiment classification via social context regularization, Neurocomputing 175 (2016): 599-609.

[39] T. Wilson, J. Wiebe, P. Hoffmann, Recognizing contextual polarity in phraselevel sentiment analysis, in Proceedings of Human Language Technologies Conference/Conference on Empirical Methods in Natural Language Processing (HLT/EMNLP 2005), Vancouver, Canada, (2005): 347354. 
[40] M.M. Bradley, P.J. Lang, Affective Norms for English Words (ANEW) Instruction Manual and Affective Ratings, Technical Report C-1, The Center for Research in Psychophysiology University of Florida, 2009.

[41] F. Nielsen, A new anew: evaluation of a word list for sentiment analysis in microblogs, in Proceedings of the ESWC2011 Workshop on 'Making Sense of Microposts': Big Things Come in Small Packages, Heraklion, Crete, Greece, 2011.

[42] A. Esuli, F. Sebastiani, Sentiwordnet: A publicly available lexical resource for opinion mining, in Proceedings of the 5th Conference on Language Resources and Evaluation, (2006): 417-422. [43] S. Baccianella, A. Esuli, F. Sebastiani, Sentiwordnet 3.0: an enhanced lexical resource for sentiment analysis and opinion mining, in Proceedings of the Seventh International Conference on Language Resources and Evaluation, Valletta, Malta, (2010): 2200-2204.

[44] M. Thelwall, K. Buckley, G. Paltoglou, Sentiment strength detection for the social web, Journal of the American Society for Information Science and Technology 63.1 (2012): 163-173.

[45] S.M. Mohammad, P. D. Turney, Crowdsourcing a word-emotion association lexicon, Computational Intelligence 29 (3) (2013): 436-465.

[46] S.M. Mohammad, S. Kiritchenko, X. Zhu, Nrc-canada: building the state-of-the art in sentiment analysis of tweets, in Proceedings of the Seventh International Workshop on Semantic Evaluation Exercises (SemEval-2013), Atlanta, Georgia, USA, 2013.

[47] E. Cambria, B. Schuller, Y. Xia, C. Havasi, New avenues in opinion mining and sentiment analysis, IEEE Intell. Syst. 28 (2) (2013): 15-21.

[48] A. Abbasi, H. Chen, A. Salem, Sentiment analysis in multiple languages: Feature selection for opinion classification in Web forums, ACM Transactions on Information Systems 26-3 (2008): 12. [49] A. S. H. Basari, B. Hussin, I. G. P. Ananta, J. Zeniarja, Opinion mining of movie review using hybrid method of support vector machine and particle swarm optimization, Procedia Engineering 53 (2013): 453-462. 
[50] X. Bai, R. Padman, E. Airoldi, Sentiment extraction from unstructured text using tabu searchenhanced markov blanket, Carnegie Mellon University, School of Computer Science,[Institute for Software Research International], 2004.

[51] A. Das, S. Bandyopadhyay, Subjectivity detection using genetic algorithm, the 1st Workshop on Computational Approaches to Subjectivity and Sentiment Analysis (WASSA10), Portugal. 2010.

[52] A. Baldominos Gómez, N. Luis Mingueza, M. C. García del Pozo, OpinAIS: An Artificial Immune System-based Framework for Opinion Mining, International Journal of Artificial Intelligence and Interactive Multimedia 3 (2015).

[53] G. Stylios, C. D. Katsis, D. Christodoulakis, Using Bio-inspired intelligence for Web opinion Mining, International Journal of Computer Applications 87.5 (2014).

[54] S. Arora, E. Mayfield, C. Penstein-Rosé, E. Nyberg, Sentiment classification using automatically extracted subgraph features, in Proceedings of the NAACL HLT 2010 Workshop on Computational Approaches to Analysis and Generation of Emotion in Text, Association for Computational Linguistics, 2010.

[55] M. Govindarajan, Sentiment Analysis of Movie Reviews using Hybrid Method of Naive Bayes and Genetic Algorithm, IJACR 3.4 (2013): 139-145.

[56] J. Carvalho, A. Prado, A. Plastino, A Statistical and Evolutionary Approach to Sentiment Analysis." Web Intelligence (WI) and Intelligent Agent Technologies (IAT), 2014 IEEE/WIC/ACM International Joint Conferences on. Vol. 2. IEEE, 2014.

[57] H. Saif, M. Fernandez, Y. He, H. Alani, Evaluation datasets for twitter sentiment analysis: a survey and a new dataset, the STS-Gold, ESSEM@AI*IA, Vol. 1096 of CEUR Workshop Proceedings, CEUR-WS.org (2013): 9-21.

[58] D. A. Shamma, L. Kennedy, E. F. Churchill, Tweet the debates: understanding community annotation of uncollected sources, Proceedings of the first SIGMM workshop on Social Media, ACM, (2009): 3-10. 
[59] M. Speriosu, N. Sudan, S. Upadhyay, J. Baldridge, Twitter polarity classification with label propagation over lexical links and the follower graph, Proceedings of the First Workshop on Unsupervised Learning in NLP, EMNLP'11, Association for Computational Linguistics, Stroudsburg, PA, USA (2011): 53-63.

[60] P. Nakov, Z. Kozareva, A. Ritter, S. Rosenthal, V. Stoyanov, T. Wilson, Semeval-2013 task 2: Sentiment analysis in twitter, In Proceedings of the 7th International Workshop on Semantic Evaluation.

[61] H. Saif, M. Fernandez, Y. He, H. Alani, On stopwords, filtering and data sparsity for sentiment analysis of Twitter, In Proceedings of the 9th language resources and evaluation conference (LREC), (2014): 810-817. 